\title{
Particle-Like Solutions of the Einstein-Dirac Equations
}

\author{
Felix Finster; Joel Smoller†, and Shing-Tung Yau \\ January 1998
}

\begin{abstract}
The coupled Einstein-Dirac equations for a static, spherically symmetric system of two fermions in a singlet spinor state are derived. Using numerical methods, we construct an infinite number of soliton-like solutions of these equations. The stability of the solutions is analyzed. For weak coupling (i.e., small rest mass of the fermions), all the solutions are linearly stable (with respect to spherically symmetric perturbations), whereas for stronger coupling, both stable and unstable solutions exist. For the physical interpretation, we discuss how the energy of the fermions and the (ADM) mass behave as functions of the rest mass of the fermions. Although gravitation is not renormalizable, our solutions of the Einstein-Dirac equations are regular and well-behaved even for strong coupling.
\end{abstract}

\section{Introduction}

In recent years, there has been much interest in the coupling of Einstein's field equations to Yang-Mills equations. In this case, the attractive gravitational force is balanced by the repulsive Yang-Mills force, and this interaction has led to many interesting and surprising results; see for example [1]-[10]. In this paper, we consider the coupling of Einstein's equations to the Dirac equation. Here the necessary repulsive mechanism is provided by the Heisenberg Uncertainty Principle.

The Einstein-Dirac equations take the form

$$
R_{j}^{i}-\frac{1}{2} R \delta_{j}^{i}=-8 \pi T_{j}^{i}, \quad(G-m) \Psi=0
$$

where $T_{j}^{i}$ is the energy-momentum tensor of the Dirac particle, $G$ denotes the Dirac operator (see [13]), and $\Psi$ is the wave function of a fermion of mass $m$. As in the abovementioned earlier studies, we consider static, spherically symmetric solutions. Since the spin of a fermion has an intrinsic orientation in space, a system consisting of a single Dirac particle cannot be spherically symmetric. In order to maintain the spherical symmetry, we are led to the study of two fermions having opposite spin; i.e. to a singlet spinor state. Of course, such a configuration does not represent a realistic physical system due to the absence of the electromagnetic interaction. More precisely, neglecting the electromagnetic interaction corresponds to the limiting case where the masses of the fermions become so large (of the order (Planck length) ${ }^{-1}$ ) that the gravitational interaction becomes the dominant force. Nevertheless, we view this study as a model problem worth considering in

\footnotetext{
${ }^{*}$ Research supported by the Deutsche Forschungsgemeinschaft and the Schweizerischer Nationalfonds.

${ }^{\dagger}$ Research supported in part by the NSF, Grant No. DMS-G-9501128.

${ }^{\ddagger}$ Research supported in part by the NSF, Grant No. 33-585-7510-2-30.
} 
order to get some understanding of the equations and their solutions. In a future publication, we will consider the more physically realistic situation where the Einstein-Dirac equations are coupled to an electromagnetic field (Maxwell's equations).

Our results are based on a certain ansatz, whereby we reduce the 4-component Dirac spinors to a 2 -component spinor system, $\Phi=(\alpha, \beta)$ with real functions $\alpha$, $\beta$. We show numerically that particle-like solutions of this type exist, both in the ground state, and in the excited states. For weak coupling, i.e. small mass $m$, the different solutions are characterized by the "rotation number", $n=0,1,2, \ldots$, of the vector $(\alpha, \beta)$ (we work in standard units $\hbar=c=\mathcal{G}=1)$. The solution with $n=0$ is the ground state, and the solutions with $n=1,2, \ldots$ describe the excited states. For small $m$, the solutions are (linearly) stable with respect to spherically symmetric perturbations. However, as $m$ gets large, several states appear for each $n$. In fact, for every $n$, the mass spectrum (i.e., the plot of the binding energy vs. the rest mass) is a spiral curve which tends to a limiting configuration. This surprising result shows that for parameter values on this limiting configuration, there are an infinite number of excited states "in the $n^{\text {th }}$ mode", while for parameter values near this limiting configuration, there are still a large, but finite number

of such excited states. Furthermore, using topological methods and bifurcation theory (see [14, part IV]), we show that in every mode, the stable solutions must become unstable as the binding energy increases. Although gravitation is not renormalizable (which means that the problem cannot be treated in a perturbation expansion), our solutions of the Einstein-Dirac equations are regular and well-behaved even for strong coupling.

\section{The Dirac Operator}

In this section, we shall derive the form of the Dirac operator in the presence of a static, spherically symmetric gravitational field. In preparation, we first give a brief mathematical introduction of the Dirac theory in curved space-time. The Dirac operator $G$ is a differential operator of first order

$$
G=i G^{j}(x) \frac{\partial}{\partial x^{j}}+B(x)
$$

where the Dirac matrices $G^{j}(x),(j=0,1,2,3)$, and $B(x)$ are $(4 \times 4)$ matrices, which depend on the space-time point $x$. The Dirac matrices and the Lorentzian metric are related by

$$
g^{j k}=\frac{1}{2}\left\{G^{j}, G^{k}\right\} \quad,
$$

where $\{.,$.$\} is the anti-commutator$

$$
\left\{G^{j}, G^{k}\right\}=\left(G^{j} G^{k}+G^{k} G^{j}\right)
$$

The basic difficulty with Dirac spinors in curved space-time is that, for a given Lorentzian metric, the Dirac matrices are not uniquely determined by the anti-commutation relations (2.2). One way of fixing the Dirac matrices is provided by the formalism of spin and frame bundles (see e.g. the first section of [11]). In this formulation, one chooses a frame $\left(u_{a}\right)_{a=0, \ldots, 3}$ and represents the Dirac matrices as linear combinations of the Dirac matrices $\gamma_{a}$ of Minkowski space,

$$
G^{j}(x)=\sum_{a=0}^{3} u_{a}^{j}(x) \gamma^{a}
$$


The matrix $B(x)$ is composed of the so-called spin connection coefficients, involving first partial derivatives of the metric and of the frame. It is quite common to choose for $\left(u_{a}\right)$ a Newman-Penrose null frame; this choice is particularly convenient for metrics of Petrov type D (see 12] for an introduction to the Newman-Penrose formalism and many applications, especially in the Kerr background). More generally, it is shown in 13 that all choices of Dirac matrices satisfying (2.2) yield unitarily equivalent Dirac operators. Furthermore, 13] gives explicit formulas for the matrix $B$ in terms of the Dirac matrices $G^{j}$. We prefer working with the formalism of [13] in the following, because it gives us more flexibility in choosing the Dirac matrices.

The wave function $\Psi$ of a Dirac particle is a solution of the Dirac equation

$$
(G-m) \Psi=0 \quad \text {. }
$$

On the wave functions, two different scalar products can be defined. In the first, we integrate the wave functions over all of space-time,

$$
<\Psi \mid \Phi>=\int \bar{\Psi} \Phi \sqrt{|g|} d^{4} x
$$

where $\bar{\Psi}=\Psi^{*}\left(\begin{array}{cc}\mathbb{1} & 0 \\ 0 & -\mathbb{1}\end{array}\right)$ is the adjoint spinor (whose definition does not depend on the gravitational field; $0, \mathbb{1}$ are $(2 \times 2)$ submatrices $)$, and $g$ denotes the determinant of the metric $g_{j k}$. The scalar product (2.4) is indefinite, but it is nevertheless useful to us because the Dirac operator is Hermitian with respect to it. The second scalar product is defined on the solutions of the Dirac equation. For this we choose a space-like hypersurface $\mathcal{H}$ together with a (future-directed) normal vector field $\nu$, and set

$$
(\Psi \mid \Phi)=\int_{\mathcal{H}} \bar{\Psi} G^{j} \Phi \nu_{j} d \mu
$$

where $d \mu$ is the invariant measure on the hypersurface $\mathcal{H}$, induced by the metric $g_{i j}$. This scalar product is positive definite, and, as a consequence of the current conservation (cf. [13])

$$
\nabla_{j} \bar{\Psi} G^{j} \Phi=0
$$

it is independent of the choice of the hypersurface $\mathcal{H}$. In direct generalization of the expression $\bar{\Psi} \gamma^{0} \Psi$ in Minkowski space (see e.g [15]), the integrand $\bar{\Psi} G^{j} \Psi \nu_{j}$ is interpreted as the probability density of the particle. This leads us to normalize solutions of the Dirac equation by requiring

$$
(\Psi \mid \Psi)=1
$$

We now return to the Dirac operator (2.1). Suppose that a 4-dimensional space-time with metric $g_{i j}$ is given. According to [13], we can choose for $G^{j}$ any $4 \times 4$ matrices which are Hermitian with respect to the scalar product (2.4) and satisfy (2.2). The matrix $B(x)$ involves first derivatives of the Dirac matrices $G^{j}$, and from [13], we have the explicit formulas

$$
B(x)=G^{j}(x) E_{j}(x)
$$

with

$$
\begin{aligned}
E_{j} & =\frac{i}{2} \rho\left(\partial_{j} \rho\right)-\frac{i}{16} \operatorname{Tr}\left(G^{m} \nabla_{j} G^{n}\right) G_{m} G_{n}+\frac{i}{8} \operatorname{Tr}\left(\rho G_{j} \nabla_{m} G^{m}\right) \rho \\
\rho & =\frac{i}{4 !} \epsilon_{i j k l} G^{i} G^{j} G^{k} G^{l}
\end{aligned}
$$


( $\epsilon_{i j k l}$ is the totally antisymmetric tensor density).

Now we will specify these formulas for the Dirac operator to static, spherically symmetric space-times. In polar coordinates $(t, r, \vartheta, \varphi)$, the metric can be written as (cf. [16, 7])

$$
\begin{aligned}
g_{i j} & =\operatorname{diag}\left(\frac{1}{T^{2}},-\frac{1}{A},-r^{2},-r^{2} \sin ^{2} \vartheta\right) \\
g^{i j} & =\operatorname{diag}\left(T^{2},-A,-\frac{1}{r^{2}},-\frac{1}{r^{2} \sin ^{2} \vartheta}\right)
\end{aligned}
$$

with volume element

$$
\sqrt{|g|}=T^{-1} A^{-\frac{1}{2}} r^{2}|\sin \vartheta| \quad,
$$

where $A=A(r)$ and $T=T(r)$ are positive functions. We shall use this form of the metric to explicitly calculate the Dirac operator (2.1). For the Dirac matrices $G^{j}(x)$, we take an ansatz as a linear combination of the usual $\gamma$-matrices in the Dirac representation

$$
\gamma^{0}=\left(\begin{array}{cc}
\mathbb{1} & 0 \\
0 & -\mathbb{1}
\end{array}\right), \quad \gamma^{i}=\left(\begin{array}{cc}
0 & \sigma^{i} \\
-\sigma^{i} & 0
\end{array}\right), \quad i=1,2,3,
$$

where $\sigma^{1}, \sigma^{2}, \sigma^{3}$ are the Pauli matrices. In order to satisfy (2.2), we must transform these Dirac matrices of the vacuum into polar coordinates and multiply them by the factors $T$ and $\sqrt{A}$,

$$
\begin{aligned}
G^{t} & =T \gamma^{0} \\
G^{r} & =\sqrt{A}\left(\gamma^{1} \cos \vartheta+\gamma^{2} \sin \vartheta \cos \varphi+\gamma^{3} \sin \vartheta \sin \varphi\right) \\
G^{\vartheta} & =\frac{1}{r}\left(-\gamma^{1} \sin \vartheta+\gamma^{2} \cos \vartheta \cos \varphi+\gamma^{3} \cos \vartheta \sin \varphi\right) \\
G^{\varphi} & =\frac{1}{r \sin \vartheta}\left(-\gamma^{2} \sin \varphi+\gamma^{3} \cos \varphi\right)
\end{aligned}
$$

This choice is convenient, because it greatly simplifies equations (2.8)-(2.10). Namely, the matrix $\rho$ becomes independent of $x$ and coincides with the usual "pseudo scalar" matrix $\gamma^{5}$ in the Dirac representation,

$$
\rho \equiv \gamma^{5}=i \gamma^{0} \gamma^{1} \gamma^{2} \gamma^{3}=\left(\begin{array}{ll}
0 & \mathbb{1} \\
\mathbb{1} & 0
\end{array}\right) \quad .
$$

As a consequence, the first and last summands in (2.9) vanish and thus

$$
\begin{aligned}
B & =-\frac{i}{16} \operatorname{Tr}\left(G^{m} \nabla_{j} G^{n}\right) G^{j} G_{m} G_{n} \\
& =-\frac{i}{16} \operatorname{Tr}\left(G^{m} \nabla_{j} G^{n}\right)\left(\delta_{m}^{j} G_{n}-\delta_{n}^{j} G_{m}+G^{j} g_{m n}+i \varepsilon_{m n p}^{j} \gamma^{5} G^{p}\right) .
\end{aligned}
$$

Using Ricci's Lemma

$$
0=4 \nabla_{j} g^{m n}=\nabla_{j} \operatorname{Tr}\left(G^{m} G^{n}\right)=\operatorname{Tr}\left(\left(\nabla_{j} G^{m}\right) G^{n}\right)+\operatorname{Tr}\left(G^{m}\left(\nabla_{j} G^{n}\right)\right)
$$

we conclude that the contributions of the first and second summands in the right bracket in (2.18) coincide and that the contribution of the third summand vanishes. Using the 
anti-symmetry of the $\epsilon$-tensor, we can, in the contribution of the fourth summand, replace the covariant derivative by a partial derivative. This gives

$$
B=\frac{i}{8} \operatorname{Tr}\left(G^{n} \nabla_{j} G^{j}\right) G_{n}+\frac{1}{16} \epsilon^{j m n p} \operatorname{Tr}\left(G_{m} \partial_{j} G_{n}\right) \gamma^{5} G_{p} \quad .
$$

The second summand in (2.19) is zero. Namely, the trace always vanishes if the tensor indices are all different,

$$
\operatorname{Tr}\left(G_{m} \partial_{j} G_{n}\right)=0 \quad \text { for } \quad m, j, n=t, r, \vartheta \text {, or } \varphi \quad \text { and } \quad m \neq j \neq n \neq m
$$

this can be verified directly using our special ansatz (2.14)-(2.17) for the Dirac matrices. In the first summand in (2.19), we can use that $\nabla_{j} G^{j}$ is a linear combination of the Dirac matrices $G^{j}$, and thus

$$
\operatorname{Tr}\left(G^{n} \nabla_{j} G^{j}\right) G_{n}=4 \nabla_{j} G^{j}
$$

We conclude that

$$
B=\frac{i}{2} \nabla_{j} G^{j}
$$

This form of $B(x)$ as a divergence of the Dirac matrices allows us to easily check that the Dirac operator is Hermitian with respect to the scalar product $<$. $\mid$. > ; indeed

$$
\begin{aligned}
<G \Psi \mid \Phi> & =\int \overline{\left(i G^{j} \frac{\partial}{\partial x^{j}}+\frac{i}{2} \nabla_{j} G^{j}\right) \Psi} \Phi \sqrt{|g|} d^{4} x \\
& =\int \bar{\Psi}\left(i G^{j} \frac{\partial}{\partial x^{j}}-\frac{i}{2} \nabla_{j} G^{j}\right) \Phi \sqrt{|g|} d^{4} x+\int \bar{\Psi}\left(i \partial_{j}\left(\sqrt{|g|} G^{j}\right)\right) \Phi d^{4} x \\
& =\int \bar{\Psi}\left(i G^{j} \frac{\partial}{\partial x^{j}}+\frac{i}{2} \nabla_{j} G^{j}\right) \Phi \sqrt{|g|} d^{4} x=<\Psi \mid G \Phi>
\end{aligned}
$$

In order to calculate the divergence (2.21), we first compute

$$
\begin{aligned}
& \frac{1}{\sqrt{|g|}} \partial_{t}\left(\sqrt{|g|} G^{t}\right)=0 \\
& \frac{1}{\sqrt{|g|}} \partial_{r}\left(\sqrt{|g|} G^{r}\right)=A^{\frac{1}{2}} T r^{-2} \partial_{r}\left(r^{2} A^{-\frac{1}{2}} T^{-1} G^{r}\right)=\left(\frac{2}{r}-\frac{T^{\prime}}{T}\right) G^{r} \\
& \frac{1}{\sqrt{|g|}} \partial_{\vartheta}\left(\sqrt{|g|} G^{\vartheta}\right) \\
& =\frac{1}{r \sin \vartheta} \partial_{\vartheta}\left(-\gamma^{1} \sin ^{2} \vartheta+\gamma^{2} \sin \vartheta \cos \vartheta \cos \varphi+\gamma^{3} \sin \vartheta \cos \vartheta \sin \varphi\right) \\
& =\frac{1}{r \sin \vartheta}\left(-2 \gamma^{1} \sin \vartheta \cos \vartheta+\gamma^{2}\left(\cos ^{2} \vartheta-\sin ^{2} \vartheta\right) \cos \varphi+\gamma^{3}\left(\cos ^{2} \vartheta-\sin ^{2} \vartheta\right) \sin \varphi\right) \\
& \frac{1}{\sqrt{|g|}} \partial_{\varphi}\left(\sqrt{|g|} G^{\varphi}\right)=\frac{1}{r \sin \vartheta}\left(-\gamma^{2} \cos \varphi-\gamma^{3} \sin \varphi\right)
\end{aligned}
$$

and thus obtain

$$
\begin{aligned}
B & =\frac{i}{2}\left(\frac{2}{r}-\frac{T^{\prime}}{T}\right) G^{r}-\frac{i}{r}\left(\gamma^{1} \cos \vartheta+\gamma^{2} \sin \vartheta \cos \varphi+\gamma^{3} \sin \vartheta \sin \varphi\right) \\
& =\frac{i}{r}\left(1-A^{-\frac{1}{2}}\right) G^{r}-\frac{i}{2} \frac{T^{\prime}}{T} G^{r} .
\end{aligned}
$$

We conclude that the Dirac operator has the form

$$
G=i G^{t} \frac{\partial}{\partial t}+G^{r}\left(i \frac{\partial}{\partial r}+\frac{i}{r}\left(1-A^{-\frac{1}{2}}\right)-\frac{i}{2} \frac{T^{\prime}}{T}\right)+i G^{\vartheta} \frac{\partial}{\partial \vartheta}+i G^{\varphi} \frac{\partial}{\partial \varphi} .
$$




\section{The Dirac Equations}

In this section, we shall separate out angular momentum from the Dirac equation (2.3) and reduce the problem to one on real 2-spinors.

We first introduce some formulas involving Pauli matrices. These will be used in this section for the separation of the angular dependence, and then, in the next section, for the computation of the energy-momentum tensor needed in Einstein's equations. We introduce the following notation:

$$
\begin{aligned}
& \sigma^{r}(\vartheta, \varphi)=\sigma^{1} \cos \vartheta+\sigma^{2} \sin \vartheta \cos \varphi+\sigma^{3} \sin \vartheta \sin \varphi \\
& \sigma^{\vartheta}(\vartheta, \varphi)=-\sigma^{1} \sin \vartheta+\sigma^{2} \cos \vartheta \cos \varphi+\sigma^{3} \cos \vartheta \sin \varphi \\
& \sigma^{\varphi}(\vartheta, \varphi)=\frac{1}{\sin \vartheta}\left(-\sigma^{2} \sin \varphi+\sigma^{3} \cos \varphi\right)
\end{aligned}
$$

These matrices are orthogonal,

$$
\operatorname{Tr}\left(\sigma^{r} \sigma^{\vartheta}\right)=\operatorname{Tr}\left(\sigma^{r} \sigma^{\varphi}\right)=\operatorname{Tr}\left(\sigma^{\vartheta} \sigma^{\varphi}\right)=0 \quad,
$$

and their square is a multiple of the identity,

$$
\left(\sigma^{r}\right)^{2}=\left(\sigma^{\vartheta}\right)^{2}=\mathbb{1}, \quad\left(\sigma^{\vartheta}\right)^{2}=\frac{\mathbb{1}}{\sin ^{2} \vartheta} \quad .
$$

Furthermore,

$$
\begin{aligned}
\sigma^{\vartheta}\left(\partial_{\vartheta} \sigma^{r}\right) & =\sigma^{\varphi}\left(\partial_{\varphi} \sigma^{r}\right)=\mathbb{1} \\
\operatorname{Tr}\left(\sigma^{\vartheta}\left(\partial_{\varphi} \sigma^{r}\right)\right) & =2 \sin \vartheta \cos \vartheta(-\cos \varphi \sin \varphi+\sin \varphi \cos \varphi)=0 \\
\operatorname{Tr}\left(\sigma^{\varphi}\left(\partial_{\vartheta} \sigma^{r}\right)\right) & =2 \frac{\cos \vartheta}{\sin \vartheta}(-\sin \varphi \cos \varphi+\cos \varphi \sin \varphi)=0
\end{aligned}
$$

In analogy to the ansatz for the Dirac spinors in the hydrogen atom for zero angular momentum (see e.g. [17]), we write the wave functions in the form

$$
\Psi_{a}=e^{-i \omega t}\left(\begin{array}{c}
u_{1} e_{a} \\
\sigma^{r} u_{2} e_{a}
\end{array}\right), \quad a=1,2 \quad,
$$

where $u_{1}(r)$ and $u_{2}(r)$ are complex-valued functions, and the $\left(e_{a}\right)_{a=1,2}$ denote the standard basis $e_{1}=(1,0), e_{2}=(0,1)$ of the two-component Pauli spinors. This ansatz is quite useful, because the Dirac equations for $\Psi_{1}$ and $\Psi_{2}$ are independent of each other,

$$
\begin{gathered}
G \Psi_{a}=\left[\left(\begin{array}{cc}
0 & \sigma^{r} \\
-\sigma^{r} & 0
\end{array}\right)\left(i \sqrt{A} \partial_{r}+\frac{i}{r}(\sqrt{A}-1)-\frac{i}{2} \frac{T^{\prime}}{T} \sqrt{A}\right)\right. \\
\left.+\omega T \gamma^{0}+\frac{2 i}{r}\left(\begin{array}{cc}
0 & \sigma^{r} \\
0 & 0
\end{array}\right)\right] \Psi_{a}
\end{gathered}
$$

where we have used (3.1). This allows us to view the Dirac equation as a two-component equation in $u_{1}, u_{2}$. In order to simplify the radial dependence, we choose new functions $\Phi_{1}$ and $\Phi_{2}$ defined by

$$
\Phi_{1}=r T^{-\frac{1}{2}} u_{1}, \quad \Phi_{2}=-i r T^{-\frac{1}{2}} u_{2} \quad,
$$


and rewrite the Dirac equation as

$$
\left[\left(\begin{array}{cc}
1 & 0 \\
0 & -1
\end{array}\right) \omega T-\left(\begin{array}{cc}
0 & 1 \\
1 & 0
\end{array}\right) \sqrt{A} \partial_{r}+\left(\begin{array}{cc}
0 & -1 \\
1 & 0
\end{array}\right) \frac{1}{r}-m\right] \Phi=0
$$

This equation is real; thus we may assume that $\Phi$ itself is real. On these two-component spinors, the scalar product $<. \mid$. $>$ takes the form

$$
<\Phi \mid \Psi>=\int_{0}^{\infty} \bar{\Phi} \Psi A^{-\frac{1}{2}} d r \quad, \quad \bar{\Phi}=\Phi \sigma^{3} \quad .
$$

The "Dirac operator" in (3.7) is Hermitian with respect to this scalar product, and the normalization condition (2.7) for the wave functions transforms into

$$
\int_{0}^{\infty}|\Phi|^{2} \frac{T}{\sqrt{A}} d r=\frac{1}{4 \pi}
$$

Finally, we write the Dirac equation as the ODE

$$
\sqrt{A} \Phi^{\prime}=\left[\omega T\left(\begin{array}{cc}
0 & -1 \\
1 & 0
\end{array}\right)+\frac{1}{r}\left(\begin{array}{cc}
1 & 0 \\
0 & -1
\end{array}\right)-m\left(\begin{array}{ll}
0 & 1 \\
1 & 0
\end{array}\right)\right] \Phi \quad .
$$

We remark that, instead of taking the ansatz (3.4) for the wave functions, we could just as well have put the matrix $\sigma^{r}$ into the upper component, i.e.

$$
\Psi_{a}=e^{-i \omega t}\left(\begin{array}{c}
\sigma^{r} u_{1} e_{a} \\
u_{2} e_{a}
\end{array}\right), \quad a=1,2 \quad .
$$

This ansatz can be reduced to (3.4) by changing the sign of the mass in the Dirac equation. To see this, we transform the wave function according to

$$
\hat{\Psi}_{a}=\gamma^{5} \Psi_{a}
$$

Then since $\hat{\Psi}$ is of the form (3.4) and satisfies the Dirac equation

$$
0=\gamma^{5}(G-m) \gamma^{5} \hat{\Psi}_{a}=-(G+m) \hat{\Psi}_{a} \quad,
$$

we can again simplify to the two-component equation (3.7). We conclude that it also makes physical sense to look at the solutions of (3.9) with negative $m$ (and positive $\omega$ ); they can be interpreted as solutions corresponding to the ansatz (3.10).

In Appendix A, we study the spinor dependence of general static, spherically symmetric solutions of the Einstein-Dirac equations, and we give a more systematic justification for the two ansatz' (3.4) and (3.10).

\section{Calculation of the Energy-Momentum Tensor}

We derive the form of the energy-momentum tensor by considering an arbitrary variation $\delta g_{i j}$ of the metric tensor. The variation of its inverse is given by $\delta g^{i j}=-g^{i k} g^{j l} \delta g_{k l}$. In order to satisfy (2.2), we vary the Dirac matrices according to

$$
\delta G^{j}=-\frac{1}{2} g^{j k}\left(\delta g_{k l}\right) G^{l}, \quad \delta G_{j}=\frac{1}{2}\left(\delta g_{j k}\right) G^{k} \quad .
$$


The energy-momentum tensor is obtained as the variation of the classical Dirac action (see e.g. [15]),

$$
S=\int \bar{\Psi}(G-m) \Psi \sqrt{|g|} d^{4} x
$$

This action is real; therefore it suffices to consider the real part of the integrand. Since the wave function $\Psi$ solves the Dirac equation for the unperturbed Dirac operator $G$, we must only consider the variation of $G$; thus

$$
\delta S=\int \operatorname{Re} \bar{\Psi}\left(i\left(\delta G^{j}\right) \frac{\partial}{\partial x^{j}}+\delta B\right) \Psi \sqrt{|g|} d^{4} x
$$

We calculate the variation of the matrix $B$ using (4.1) and (2.8), (2.9),

$$
\begin{aligned}
\operatorname{Re} \bar{\Psi} \delta B \Psi & =\frac{1}{16} \operatorname{Im} \delta\left(\operatorname{Tr}\left(G^{m} \nabla_{j} G^{n}\right) \bar{\Psi} G^{j} G_{m} G_{n} \Psi\right) \\
& =\frac{1}{16} \delta\left(\operatorname{Tr}\left(G^{m} \nabla_{j} G^{n}\right) \operatorname{Im}\left(\bar{\Psi} G^{j} G_{m} G_{n} \Psi\right)\right) \\
& =\frac{1}{16} \delta\left(\epsilon^{j m n p} \operatorname{Tr}\left(G_{m} \nabla_{j} G_{n}\right) \bar{\Psi} \gamma^{5} G_{p} \Psi\right) \\
& =\frac{1}{16} \delta\left(\epsilon^{j m n p} \operatorname{Tr}\left(G_{m} \partial_{j} G_{n}\right) \bar{\Psi} \gamma^{5} G_{p} \Psi\right) \\
& \stackrel{(2.20)}{=} \frac{1}{16} \epsilon^{j m n p} \delta \operatorname{Tr}\left(G_{m} \partial_{j} G_{n}\right) \bar{\Psi} \gamma^{5} G_{p} \Psi \\
& =\frac{1}{16} \epsilon^{j m n p}\left(\delta g_{m k}\right) \operatorname{Tr}\left(G^{k} \partial_{j} G_{n}\right) \bar{\Psi} \gamma^{5} G_{p} \Psi
\end{aligned}
$$

Notice that the trace in the last equation does not necessarily vanish. But we can use the fact that we are dealing with a spin singlet; this implies that the expectation value of the pseudovector $\gamma^{5} G_{p}$ is zero,

$$
\sum_{a=1}^{2} \overline{\Psi_{a}} \gamma^{5} G_{p} \Psi_{a}=0
$$

(this can be checked by a short explicit calculation). Thus we only have to consider the variation $\delta G^{j}$ of the Dirac matrices in (4.2). We substitute (4.1) into (4.2) and obtain for the spin singlet

$$
\delta S=\int \frac{1}{2} \sum_{a=1}^{2} \operatorname{Re} \overline{\Psi_{a}}\left(i G_{j} \frac{\partial}{\partial x^{k}}\right) \Psi_{a} \delta g^{j k} \sqrt{|g|} d^{4} x
$$

Thus the energy-momentum tensor has the form

$$
T_{j k}=\frac{1}{2} \sum_{a=1}^{2} \operatorname{Re} \overline{\Psi_{a}}\left(i G_{j} \frac{\partial}{\partial x^{k}}+i G_{k} \frac{\partial}{\partial x^{j}}\right) \Psi_{a}
$$

We compute

$$
\begin{aligned}
& \operatorname{Re} \sum_{a=1}^{2} \overline{\Psi_{a}} i G^{t} \partial_{t} \Psi_{a}=2 \omega T|\Psi|^{2}=2 \omega T^{2} r^{-2}|\Phi|^{2} \\
& \operatorname{Re} \sum_{a=1}^{2} \overline{\Psi_{a}} i G^{t} \partial_{r} \Psi_{a}=\operatorname{Re} 2 i T\left(r^{-1} \sqrt{T} \Phi\right) \partial_{r}\left(r^{-1} \sqrt{T} \Phi\right)=0
\end{aligned}
$$




$$
\begin{aligned}
& \operatorname{Re} \sum_{a=1}^{2} \overline{\Psi_{a}} i G^{r} \partial_{t} \Psi_{a}=\operatorname{Re} \omega \sum_{a=1}^{2} \overline{\Psi_{a}} G^{r} \Psi_{a}=\operatorname{Re} 2 i \omega \frac{\sqrt{A} T}{r^{2}} \Phi\left(\begin{array}{cc}
0 & 1 \\
-1 & 0
\end{array}\right) \Phi=0 \\
& \operatorname{Re} \sum_{a=1}^{2} \overline{\Psi_{a}} i G^{r} \partial_{r} \Psi_{a}=\operatorname{Re} \sum_{a=1}^{2} i \sqrt{A} \overline{\Psi_{a}}\left(\begin{array}{cc}
0 & \mathbb{1} \\
-\mathbb{1} & 0
\end{array}\right) \frac{\vec{x} \vec{\sigma}}{r} \partial_{r} \Psi_{a} \\
& =\operatorname{Re} 2 i \sqrt{A}\left(r^{-1} \sqrt{T} \Phi\right)\left(\begin{array}{cc}
0 & i \\
-i & 0
\end{array}\right) \partial_{r}\left(r^{-1} \sqrt{T} \Phi\right) \\
& =\operatorname{Re} 2 \sqrt{A} T r^{-2} \Phi\left(\begin{array}{cc}
0 & -1 \\
1 & 0
\end{array}\right) \partial_{r} \Phi \\
& \text { (3.9) }-2 \omega T^{2} r^{-2}|\Phi|^{2}+4 T r^{-3} \Phi_{1} \Phi_{2}+2 m T r^{-2}\left(\Phi_{1}^{2}-\Phi_{2}^{2}\right) \\
& \operatorname{Re} \sum_{a=1}^{2} \overline{\Psi_{a}} i G^{\vartheta} \partial_{\vartheta} \Psi_{a}=\operatorname{Re} r^{-1} \sum_{a=1}^{2} \overline{\Psi_{a}}\left(\begin{array}{cc}
0 & i \mathbb{1} \\
-i \mathbb{1} & 0
\end{array}\right) \sigma^{\vartheta} \partial_{\vartheta} \Psi_{a} \\
& \text { (3.1) }-2 r^{-3} T \Phi_{1} \Phi_{2} \\
& \operatorname{Re} \sum_{a=1}^{2} \overline{\Psi_{a}} i G^{\varphi} \partial_{\varphi} \Psi_{a}=\operatorname{Re} r^{-1} \sum_{a=1}^{2} \overline{\Psi_{a}}\left(\begin{array}{cc}
0 & i \mathbb{1} \\
-i \mathbb{1} & 0
\end{array}\right) \sigma^{\varphi} \partial_{\varphi} \Psi_{a} \\
& \text { (3.1) }-2 r^{-3} T \Phi_{1} \Phi_{2} \text {. }
\end{aligned}
$$

All other combinations of the indices vanish because of (3.2), (3.3), and the orthogonality of $\sigma^{r}, \sigma^{\vartheta}, \sigma^{\varphi}$. We conclude that

$$
\begin{gathered}
T_{j}^{i}=r^{-2} \operatorname{diag}\left(2 \omega T^{2}|\Phi|^{2},-2 \omega T^{2}|\Phi|^{2}+4 T r^{-1} \Phi_{1} \Phi_{2}+2 m T\left(\Phi_{1}^{2}-\Phi_{2}^{2}\right),\right. \\
\left.-2 T r^{-1} \Phi_{1} \Phi_{2},-2 T r^{-1} \Phi_{1} \Phi_{2}\right)
\end{gathered}
$$

As a check, we calculate the trace of $T_{j}^{i}$ directly from (4.4),

$$
\begin{aligned}
T_{j}^{j} & =\sum_{a=1}^{2} \operatorname{Re}\left(\overline{\Psi_{a}}\left(i G^{j} \partial_{j}\right) \Psi_{a}\right)=\sum_{a=1}^{2} \operatorname{Re}\left(\overline{\Psi_{a}}\left(i G^{j} \partial_{j}+B\right) \Psi_{a}\right) \\
& =m \sum_{a=1}^{2} \overline{\Psi_{a}} \Psi_{a}=2 m \operatorname{Tr}^{-2}\left(\Phi_{1}^{2}-\Phi_{2}^{2}\right)
\end{aligned}
$$

and we see that it agrees with taking the trace of (4.5).

\section{The Field Equations}

The Einstein Tensor $G_{j}^{i}=R_{j}^{i}-\frac{1}{2} R \delta_{j}^{i}$ has the form (see e.g. [16])

$$
\begin{aligned}
& G_{0}^{0}=-\frac{1}{r^{2}}+\frac{A}{r^{2}}+\frac{A^{\prime}}{r} \\
& G_{1}^{1}=-\frac{1}{r^{2}}+\frac{A}{r^{2}}-\frac{2 A T^{\prime}}{r T} \\
& G_{2}^{2}=G_{3}^{3}=\frac{A^{\prime}}{2 r}-\frac{A T^{\prime}}{r T}-\frac{A^{\prime} T^{\prime}}{2 T}+\frac{2 A T^{\prime 2}}{T^{2}}-\frac{A T^{\prime \prime}}{T}
\end{aligned}
$$


with all other components vanishing. Thus, using (4.5), Einstein's field equations $G_{j}^{i}=$ $-8 \pi T_{j}^{i}$ become

$$
\begin{gathered}
-16 \pi \omega T^{2}|\Phi|^{2}=r A^{\prime}-(1-A) \\
-16 \pi \omega T^{2}|\Phi|^{2}+32 \pi r^{-1} T \Phi_{1} \Phi_{2}+16 \pi m T\left(\Phi_{1}^{2}-\Phi_{2}^{2}\right)=2 r A \frac{T^{\prime}}{T}+(1-A) \\
-16 \pi T r^{-1} \Phi_{1} \Phi_{2}=A\left[r^{2} \frac{T^{\prime \prime}}{T}+r^{2} \frac{A^{\prime} T^{\prime}}{2 A T}-2 r^{2}\left(\frac{T^{\prime}}{T}\right)^{2}-r \frac{A^{\prime}}{2 A}+r \frac{T^{\prime}}{T}\right] .
\end{gathered}
$$

Using the notation $\alpha=\Phi_{1}, \beta=\Phi_{2}$, the equations (3.9), (5.1), and (5.2) can be written as

$$
\begin{aligned}
\sqrt{A} \alpha^{\prime} & =\frac{1}{r} \alpha-(\omega T+m) \beta \\
\sqrt{A} \beta^{\prime} & =(\omega T-m) \alpha-\frac{1}{r} \beta \\
r A^{\prime} & =1-A-16 \pi \omega T^{2}\left(\alpha^{2}+\beta^{2}\right) \\
2 r A \frac{T^{\prime}}{T} & =A-1-16 \pi \omega T^{2}\left(\alpha^{2}+\beta^{2}\right)+32 \pi \frac{1}{r} T \alpha \beta+16 \pi m T\left(\alpha^{2}-\beta^{2}\right) .
\end{aligned}
$$

By direct computation (or, e.g. by using Mathematica), we see that equation (5.3) is implied by this set of equations. It is sometimes useful to rewrite the Dirac equations (5.4) and (5.5) in matrix notation as

$$
\sqrt{A} \Phi^{\prime}=\left(\begin{array}{cc}
1 / r & -\omega T-m \\
\omega T-m & -1 / r
\end{array}\right) \Phi \quad .
$$

The normalization condition (2.7) takes the form

$$
\int_{0}^{\infty}|\Phi|^{2} \frac{T}{\sqrt{A}} d r=\frac{1}{4 \pi}
$$

If we assume regularity of the solution at $r=0$, we obtain the following Taylor series expansions

$$
\begin{aligned}
\alpha(r) & =\alpha_{1} r+\mathcal{O}\left(r^{3}\right) \\
\beta(r) & =\frac{1}{3}\left(\omega T_{0}-m\right) \alpha_{1} r^{2}+\mathcal{O}\left(r^{3}\right) \\
A(r) & =1-\frac{2}{3} \omega T_{0}^{2} \alpha_{1}^{2} r^{2}+\mathcal{O}\left(r^{3}\right) \\
T(r) & =T_{0}-\frac{m}{6}\left(4 \omega T_{0}-3 m\right) T_{0}^{2} \alpha_{1}^{2} r^{2}+\mathcal{O}\left(r^{3}\right)
\end{aligned}
$$

where $\alpha_{1}, T_{0}, \omega$ and $m$ are (real) parameters. We also require that our solutions have finite $(\mathrm{ADM})$ mass; namely

$$
\rho:=\lim _{r \rightarrow \infty} \frac{r}{2}(1-A(r))<\infty \quad .
$$

This implies that

$$
\lim _{r \rightarrow \infty} A(r)=1
$$

Finally, in order that the metric be asymptotically Minkowskian, we require that

$$
\lim _{r \rightarrow \infty} T(r)=1
$$




\section{Scaling of the Equations}

For the numerical computations, it is difficult to take into account condition (5.15) and the integral condition (5.8). Therefore we find it convenient to temporarily replace these constraints by the weaker conditions

$$
\lim _{r \rightarrow \infty} T(r)<\infty \quad \text { and } \quad \int_{0}^{\infty}|\Phi|^{2} \frac{T}{\sqrt{A}} d r<\infty
$$

and instead set

$$
T_{0}=1 \quad \text { and } \quad m= \pm 1 \quad .
$$

This is justified by the following scaling argument which shows that there is a one-to-one correspondence between solutions satisfying (6.1), (6.2) and solutions satisfying (5.15) and (5.8).

Thus, suppose we have a solution $(\alpha, \beta, T, A)$ of (5.4)-(5.7), (5.13) satisfying (6.1), (6.2). Then, with the parameters $\lambda$ and $\tau$ defined by

$$
\begin{aligned}
\lambda & =\left(4 \pi \int_{0}^{\infty}\left(\alpha^{2}+\beta^{2}\right) \frac{T}{\sqrt{A}} d r\right)^{\frac{1}{2}} \\
\tau & =\lim _{r \rightarrow \infty} T(r)
\end{aligned}
$$

we introduce the new functions

$$
\begin{aligned}
\tilde{\alpha}(r) & =\sqrt{\frac{\tau}{\lambda}} \alpha(\lambda r) \\
\tilde{\beta}(r) & =\sqrt{\frac{\tau}{\lambda}} \beta(\lambda r) \\
\tilde{A}(r) & =A(\lambda r) \\
\tilde{T}(r) & =\tau^{-1} T(\lambda r)
\end{aligned}
$$

A direct calculation shows that these functions satisfy the equations (5.4)-(5.7) with $m, \omega$ replaced by

$$
\tilde{m}=\lambda m, \quad \tilde{w}=\lambda \omega \tau \quad .
$$

Moreover, it is easy to check that

$$
\begin{aligned}
\int_{0}^{\infty}\left(\tilde{\alpha}^{2}+\tilde{\beta}^{2}\right) \frac{\tilde{T}}{\sqrt{\tilde{A}}} d r & =\frac{1}{4 \pi} \\
\lim _{r \rightarrow \infty} \tilde{T}(r) & =1
\end{aligned}
$$

and $\lim _{r \rightarrow \infty} \frac{r}{2}(1-\tilde{A}(r))<\infty$. We note that, as long as $\lambda, \tau$ are positive and non-zero, the transformation from the un-tilde variables to the tilde variables is one-to-one.

Finally, we point out that for the physical interpretation, the conditions (5.8) and (5.15) must hold and hence only the scaled solutions can have physical significance.

\section{Existence and Properties of the Solutions}

Using the local Taylor expansion about $r=0$, (5.9)-(5.12), and setting $T(0)=1$ and $m= \pm 1$, we construct initial data at $r=10^{-5}$ and then use the standard Mathematica 
ODE solver. We shoot for numerical solutions by fixing $\alpha_{1}$ and varying $\omega$. Using accuracy $10^{-8}$, we found that solutions varied continuously with $\alpha_{1}$, (and $\omega$ ), indicating that this procedure is well-behaved. The solutions we find satisfy

$$
\begin{aligned}
\lim _{r \rightarrow \infty} r^{2}\left(\alpha(r)^{2}+\beta(r)^{2}\right) & =0, & & \lim _{r \rightarrow \infty} A(r)=1 \\
\lim _{r \rightarrow \infty} \frac{r}{2}(1-A(r)) & <\infty, & & \lim _{r \rightarrow \infty} T(r)=\tau>0,
\end{aligned}
$$

and, in addition, $T$ and $A$ stay positive for all $r$. In order to fulfill the important other two conditions (5.8),(5.15), we merely scale our variables in the manner described in section 6 .

For any given $\alpha_{1}>0$, we found a unique ground state corresponding to the parameter value $\omega_{0}$, together with a countable number of distinct excited states corresponding to parameter values $\omega_{n}, n=1,2, \ldots$, where $\omega_{0}<\omega_{1}<\ldots<\omega_{\max }\left(\alpha_{1}\right)$. For $\alpha_{1}=0.02$ and $m=1$, the scaled solutions for the ground state and for the first and second excited states are depicted in Figures 1, 2 and 迎. These solutions have the following general characteristics: The graphs of the functions $T(r)$ have the same qualitative form for all values $\omega_{n}$. The functions $A(r)$, however, have their graphs changing with $\omega_{n}$; indeed, for the $n$th excited state, the graph of $A$ has precisely $n+1$ minima. The tangent vector to the curve $(\alpha(r), \beta(r))$ for large $r$ lies alternately in either the first or third quadrants.

For larger values of $\alpha_{1}$ (and $m=1$ ), the solutions have a similar form, but the $\alpha-\beta$ graphs of the ground state have self-intersections and are thus of a different shape. This is illustrated for three different values of $\alpha_{1}$ in Figure 4 . We found that for the ground state,

$$
\lim _{\alpha_{1} \rightarrow \infty} T_{0}\left(\alpha_{1}\right)=\infty \quad \text { and } \quad \lim _{\alpha_{1} \rightarrow 0} T_{0}\left(\alpha_{1}\right)=1
$$

Moreover, in every case, $T$ is a monotone decreasing function of $r$.

We also obtained solutions for $m=-1$ by using similar methods. The results are qualitatively the same with the exception that, in this case, the tangent vector to the $(\alpha, \beta)$ curve for large $r$ lies alternatively in quadrants two and four, see Figures 5 and 6 .

The mass and the energy of the solutions we found have some interesting and surprising features, which we shall now describe. We consider the fixed $n^{\text {th }}$ excited state and, by varying $\alpha_{1}$ and adjusting $\omega$ (for fixed $m= \pm 1$ ), we obtain a one-parameter family of solutions (parametrized e.g. by $\alpha_{1}$ ). After scaling, we find that solutions can only exist for a bounded range of $m$. For every value of $m$ in this range, we obtain an at most countable number of solutions, which can be characterized by two physical parameters: the energy $\omega$ of the fermions, and the (ADM) mass (5.13). We find that we always have $\omega<|m|$, which means that the fermions are in a bound state. If we plot the binding energy $\omega-|m|$ vs. the mass $m$ (i.e., the mass-energy spectrum), we find that this curve is a spiral which tends to a limiting configuration. This is shown in Figure 0 for the ground state $(\mathrm{G})$, the first and second excited states $(\mathrm{F}, \mathrm{S})$, and the lowest and next excited states for negative mass (L, $\mathrm{N}$ ). The spirals seem to have a self-similarity; this is illustrated in Figure 8 (we stopped the computation when the limitations of our numerics were reached). The (ADM) mass $\rho$ can be viewed as the total energy of both the gravitational field and the fermions. Thus the quantity $\rho-2 \omega$ gives the energy of the gravitational field. As is shown in Figure 9, it is always positive and, if plotted versus $m$, gives curves which again look like spirals. Furthermore, one can ask how much energy is gained (or needed) to form the singlet state.

\footnotetext{
${ }^{1}$ We point out that we also plot the scaled variables in all the other figures.
} 
For this, we must compare the (ADM) mass with the total rest mass $2|m|$; i.e., the energy of two fermions at rest which are so much delocalized in space that their gravitational interaction becomes zero. This is plotted in Figure 10. For small $m$, the function $\rho-2|m|$ is negative, which shows that one gains energy by forming the singlet state. (This gives a first hint that these states might be stable, because energy is needed to break up the binding.) For large values of $m$, however, (more precisely, shortly after the "turning point" of $m$; see Figure 10), $\rho-2|m|$ becomes positive. This indicates that the solutions should no longer be stable. This is indeed true and will be shown in the next section.

We note that our scaling technique is essential for obtaining the mass spectrum - the unscaled variables do not have "spirals".

\section{Stability of the Solutions}

In this section, we shall consider the stability of our solutions under spherically symmetric perturbations. To this end, we consider the spherically symmetric, time-dependent Lorentzian metric of the form

$$
d s^{2}=T^{-2}(r, t) d t^{2}-A^{-1}(r, t) d r^{2}-r^{2}\left(d \vartheta^{2}+\sin ^{2} \vartheta d \varphi^{2}\right) \quad .
$$

The time-dependent Dirac operator can again be calculated using (2.21). One gets the following generalization of (2.22) (the dot denotes t-derivatives):

$$
G=G^{t}\left(i \frac{\partial}{\partial t}-\frac{i}{4} \frac{\dot{A}}{A}\right)+G^{r}\left(i \frac{\partial}{\partial r}+\frac{i}{r}\left(1-A^{-\frac{1}{2}}\right)-\frac{i}{2} \frac{T^{\prime}}{T}\right)+i G^{\vartheta} \partial_{\vartheta}+i G^{\varphi} \partial_{\varphi} \quad .
$$

As in Section 3, we separate out the angular momentum by setting (cf. (3.4))

$$
\Psi=\frac{\sqrt{T}}{r}\left(\begin{array}{c}
z_{1}(r, t) e_{a} \\
i \sigma^{r} z_{2}(r, t) e_{a}
\end{array}\right) \quad, \quad a=1,2
$$

This gives the two-component, time-dependent Dirac equation

$$
\begin{aligned}
& {\left[\left(\begin{array}{cc}
1 & 0 \\
0 & -1
\end{array}\right)\left(i T \partial_{t}-\frac{i}{4} T \frac{\dot{A}}{A}+\frac{i}{2} \dot{T}\right)\right.} \\
& \left.-\left(\begin{array}{cc}
0 & 1 \\
1 & 0
\end{array}\right) \sqrt{A} \partial_{r}+\left(\begin{array}{cc}
0 & -1 \\
1 & 0
\end{array}\right) \frac{1}{r}-m\right]\left(\begin{array}{l}
z_{1} \\
z_{2}
\end{array}\right)=0
\end{aligned}
$$

Observe that, in contrast to (3.7), this equation is complex, and thus we cannot assume here that the spinors are real. Calculation of the energy-momentum tensor and the Einstein tensor gives the equations

$$
\begin{aligned}
-\frac{1}{r^{2}}+\frac{A}{r^{2}}+\frac{A^{\prime}}{r} & =-8 \pi \frac{2 i T^{2}}{r^{2}} \operatorname{Re}\left(\overline{z_{1}} \partial_{t} z_{1}+\overline{z_{2}} \partial_{t} z_{2}\right) \\
\frac{T^{2} \dot{A}}{r A} & =-8 \pi \operatorname{Re}\left(\frac{i T^{2}}{r^{2}}\left(\overline{z_{1}} \partial_{r} z_{1}+\overline{z_{2}} \partial_{r} z_{2}\right)+\frac{T^{3} A^{-\frac{1}{2}}}{r^{2}}\left(\overline{z_{1}} \partial_{t} z_{2}-\overline{z_{2}} \partial_{t} z_{1}\right)\right) \\
-\frac{1}{r^{2}}+\frac{A}{r^{2}}-\frac{2 A T^{\prime}}{r T} & =8 \pi \frac{2 T A^{\frac{1}{2}}}{r^{2}} \operatorname{Re}\left(\overline{z_{1}} \partial_{r} z_{2}-\overline{z_{2}} \partial_{r} z_{1}\right) \\
\frac{A^{\prime}}{2 r}-\frac{A T^{\prime}}{r T}-\frac{A^{\prime} T^{\prime}}{2}+ & \frac{2 A T^{\prime}}{T^{2}}-\frac{A T^{\prime \prime}}{T}-\frac{3 T^{2} \dot{A}^{2}}{4 A^{2}}+\frac{T \dot{A} \dot{T}}{2 A}+\frac{T^{2} \ddot{A}}{2 A}=8 \pi \frac{2}{r^{3}} T \operatorname{Re}\left(\overline{z_{1}} z_{2}\right)
\end{aligned}
$$


Suppose that a solution $(\alpha(r), \beta(r), A(r), T(r))$ of the equations (5.4)-(5.7) and (5.8), (5.14), (5.15) is given for some values of the parameters $m, \omega$. Before making an ansatz for a time-dependent perturbation of this solution, it is convenient to introduce new spinor variables $\alpha(r, t), \beta(r, t)$ by setting

$$
z_{1}(r, t)=e^{-i \omega t} \alpha(r, t) \quad, \quad z_{2}(r, t)=e^{-i \omega t} \beta(r, t) \quad .
$$

This has the advantage that we get the ansatz (3.4) simply by assuming that $\alpha$ and $\beta$ are time independent. We consider spherically symmetric, time-dependent perturbations of the form

$$
\begin{aligned}
\alpha(r, t) & =\alpha(r)+\varepsilon\left(a_{1}(r, t)+i a_{2}(r, t)\right) \\
\beta(r, t) & =\beta(r)+\varepsilon\left(b_{1}(r, t)+i b_{2}(r, t)\right) \\
A(r, t) & =A(r)+\varepsilon A_{1}(r, t) \\
T(r, t) & =T(r)+\varepsilon T_{1}(r, t)
\end{aligned}
$$

where we look at the real and imaginary parts separately ( $a_{j}$ and $b_{j}$ are real functions). Substituting into the Einstein-Dirac equations and only considering the first order terms in $\varepsilon$ gives (using Mathematica) a system of linear differential equations. If these equations admitted solutions with an exponentially growing time dependence, the original solution would be unstable. Therefore we separate out the time dependence by writing

$$
\begin{array}{rlrl}
a_{j}(r, t) & =e^{\kappa t} a_{j}(r), & & b_{j}(r, t)=e^{\kappa t} b_{j}(r), j=1,2, \\
A_{1}(r, t)=e^{\kappa t} A_{1}(r), & T_{1}(r, t)=e^{\kappa t} T_{1}(r)
\end{array} .
$$

This gives the following system of five ordinary differential equations

$$
\begin{aligned}
\sqrt{A} a_{1}^{\prime}= & \frac{a_{1}}{r}-(m+\omega T) b_{1}+\kappa T b_{2}-\frac{A_{1}}{2 A}\left(\frac{\alpha}{r}-(m+\omega T) \beta\right)-\omega T_{1} \beta \\
\sqrt{A} a_{2}^{\prime}= & \frac{a_{2}}{r}-(m+\omega T) b_{2}-\kappa T b_{1}+\kappa \frac{A_{1}}{4 A} T \beta-\kappa \frac{T_{1}}{2} \beta \\
\sqrt{A} b_{1}^{\prime}= & -(m-\omega T) a_{1}-\frac{b_{1}}{r}-\kappa T a_{2}-\frac{A_{1}}{2 A}\left(-(m-\omega T) \alpha-\frac{\beta}{r}\right)+\omega T_{1} \alpha \\
\sqrt{A} b_{2}^{\prime}= & -(m-\omega T) a_{2}-\frac{b_{2}}{r}+\kappa T a_{1}-\kappa \frac{A_{1}}{4 A} T \alpha+\kappa \frac{T_{1}}{2} \alpha \\
2 r A T_{1}^{\prime}= & \frac{A_{1} T}{A}-T_{1}+A T_{1}+\frac{32 \pi T^{2}}{r}\left(a_{1} \beta+b_{1} \alpha\right) \\
& +16 \pi T^{2} \alpha\left(2 m a_{1}-2 \omega T a_{1}+\kappa T a_{2}\right)-16 \pi T^{2} \beta\left(2 m b_{1}+2 \omega T b_{1}-\kappa T b_{2}\right) \\
& -16 \pi T_{1}\left(3 \omega T^{2}\left(\alpha^{2}+\beta^{2}\right)-\frac{4}{r} T \alpha \beta-2 m T\left(\alpha^{2}-\beta^{2}\right)\right) \\
& +16 \pi \frac{A_{1} T}{A}\left(w T^{2}\left(\alpha^{2}+\beta^{2}\right)-\frac{2}{r} T \alpha \beta-m T\left(\alpha^{2}-\beta^{2}\right)\right)
\end{aligned}
$$

together with the algebraic equation

$$
A_{1}=16 \pi \frac{\sqrt{A} T}{\kappa r}\left(-\left(\kappa b_{1}+2 \omega b_{2}\right) \alpha+\left(\kappa a_{1}+2 \omega a_{2}\right) \beta\right)
$$

The consistency of these equations (i.e., that the equation $G_{2}^{2}=-8 \pi T_{2}^{2}$ is identically satisfied) was again checked with Mathematica. We want to show that there are no solutions for $\kappa>0$; this implies stability. 
The above equations come with initial conditions at $r=0$ and additional constraints, which we will now describe. A Taylor expansion about $r=0$ gives, similar to (5.9)-(5.12), the following expansions near $r=0$

$$
\begin{aligned}
& a_{1}(r)=a_{10} r+\mathcal{O}\left(r^{2}\right), \quad a_{2}(r)=a_{20} r+\mathcal{O}\left(r^{2}\right) \\
& b_{1}(r)=\mathcal{O}\left(r^{2}\right) \quad, \quad b_{2}(r)=\mathcal{O}\left(r^{2}\right) \\
& A_{1}(r)=\mathcal{O}\left(r^{2}\right) \quad, \quad T_{1}(r)=T_{10}+\mathcal{O}\left(r^{2}\right) \quad .
\end{aligned}
$$

We have three parameters $a_{10}, a_{20}$ and $T_{10}$ to characterize the solutions. Since the metric must be asymptotically Minkowskian, we demand moreover that

$$
\begin{aligned}
& \lim _{r \rightarrow \infty} A_{1}(r)=0 \\
& \lim _{r \rightarrow \infty} T_{1}(r)=0
\end{aligned}
$$

Furthermore, the wave functions must be normalized, which means that (cf. (3.8)),

$$
\int_{0}^{\infty}\left(\alpha^{2}(r, t)+\beta^{2}(r, t)\right) \frac{T(r, t)}{\sqrt{A(r, t)}} d r=\frac{1}{4 \pi} \quad,
$$

for all $t$. This time-dependent normalization condition appears to make the analysis very complicated. It turns out, however, that we do not have to consider it at all, because, for the perturbation (8.6), (8.7), it is automatically satisfied.

To see this, note that, as a consequence of the current conservation (2.6), the normalization integral (8.19) is actually time-independent. But, in the limit $t \rightarrow-\infty$, the time-dependent solution (8.2)-(8.5) of the Einstein-Dirac equations goes over into the static, unperturbed solution $(\alpha, \beta, A, T)$, and thus (8.19) holds in this limit. It follows that (8.19) holds for all $t$. In other words, the linear contribution in $\varepsilon$ to (8.19) vanishes as a consequence of the linearized Einstein-Dirac equations (8.8)-(8.12), (8.13). We must only make sure that the integral (8.19) is finite for all $t$.

These conditions can be simplified. To see this, we first consider the infinitesimal time reparametrization

$$
t \rightarrow t-\varepsilon \frac{T_{1}(r=0)}{\kappa T(r=0)} e^{\kappa t} \quad .
$$

This transformation does not destroy the ansatz (8.2)-(8.7); it only changes the functions $a_{2}, b_{2}$ and $T_{1}$ according to

$$
\begin{aligned}
& T_{1}(r) \quad \rightarrow \quad T_{1}(r)-T_{1}(0) \frac{T(r)}{T(0)} \\
& a_{2}(r) \quad \rightarrow \quad a_{2}(r)-\frac{\omega}{\kappa} \frac{T_{1}(0)}{T(0)} \alpha(r) \\
& b_{2}(r) \quad \rightarrow \quad b_{2}(r)-\frac{\omega}{\kappa} \frac{T_{1}(0)}{T(0)} \beta(r)
\end{aligned}
$$

Thus we can arrange that $T_{1}$ vanishes at the origin,

$$
T_{1}(r)=\mathcal{O}(r) \quad,
$$

provided that 8.18 ) is replaced by the weaker condition

$$
\lim _{r \rightarrow \infty} T_{1}(r)=\mu \quad \text { for some } \mu, 0<\mu<\infty \quad .
$$


This makes the numerics easier, because we now have only two free parameters $a_{10}, a_{20}$ at the origin to characterize the solution. Furthermore, using the linearity of the equations, we can multiply every solution by a (non-zero) arbitrary real number. This allows us to fix one of the parameters (e.g. by setting $a_{20}=1$ ), and thus we end up with only one free parameter.

Our strategy is to show that, for any $\kappa>0$, there are no solutions for which the normalization integral (8.19) is finite; this will imply stability. In order to explain the technique and the difficulty for the numerics, we consider Figure 11, where typical plots of $\left(a_{1}, b_{1}\right),\left(a_{2}, b_{2}\right)$ for a small value of $\kappa$, and the ground state solution of Figure 1, are shown. According to 8.14, 8.15), both the $\left(a_{1}, b_{1}\right)$ and the $\left(a_{2}, b_{2}\right)$ curves start at the origin. We want to show that at least one of these curves stays bounded away from the origin as $r \rightarrow \infty$, no matter how we choose $\kappa$ and the initial values $a_{10}, a_{20}$. This will imply that the integral (8.19) is unbounded. Figure 11 is interesting because it almost looks as if this happened: the $\left(a_{2}, b_{2}\right)$ curve looks like the $\alpha-\beta$-plot of the ground state, whereas $\left(a_{1}, b_{1}\right)$ is almost like the $\alpha-\beta$-plot of the first excited state. The $\left(a_{1}, b_{1}\right)$ curve is not quite closed, however, and it is difficult to decide whether this is just an artifact of the numerics or whether it actually means that there is no normalizable solution. The numerics are especially delicate because, for small values of $\kappa$, the values of $\left(a_{2}, b_{2}\right)$ are much larger than $\left(a_{1}, b_{1}\right)$ (notice the different scales on the plots of Figure 11). In order to improve the accuracy of the numerics, it is useful to note that, numerically, $a_{2}$ and $b_{2}$ are, to a very good approximation, multiples of the unperturbed wave functions $\alpha, \beta$, i.e. $a_{2} \approx \mu \alpha, b_{2} \approx \mu \beta$ for some real constant $\mu$. We can eliminate this dominant contribution to $a_{2}, b_{2}$ by introducing new variables

$$
\hat{a}_{2}=a_{2}-\mu \alpha, \quad \hat{b}_{2}=b_{2}-\mu \beta
$$

and rewriting our ODEs in the functions $\left(a_{1}, b_{1}, \hat{a}_{2}, \hat{b}_{2}, A_{1}, T_{1}\right)$; this gives a system of five inhomogeneous, linear equations. From these, we obtain the plots in Figure 12. The $\left(a_{1}, b_{1}\right)$-curve is similar to that of Figure 11; the $\left(\hat{a}_{2}, \hat{b}_{2}\right)$-plot, however, gives a much more detailed view of the imaginary part of $\alpha(r, t), \beta(r, t)$ (notice again the different scales on the plot).

Next we describe our method to determine the initial data at $r=0$ for the solutions (i.e. the parameters $a_{10}, a_{20}$, for the original equations (8.8)-(8.12)). It turns out that the integral (8.19) is only finite for all $t$ if both $\left(a_{1}, b_{1}\right)$ and $\left(a_{2}, b_{2}\right)$ tend to zero for large $r$; indeed, from the numerics we see that $\left(a_{1}, b_{1}\right)$ and $\left(a_{2}, b_{2}\right)$ cannot have infinite oscillations. It is an efficient technique to fix the initial values by trying to satisfy the first part of these conditions

$$
\lim _{r \rightarrow \infty}\left(a_{1}(r), b_{1}(r)\right)=(0,0) \quad .
$$

This can be done by varying the initial values in such a way that

$$
\left(a_{1}(R)^{2}+a_{2}(R)^{2}\right) \rightarrow \min \quad,
$$

where $R$ is the value of $r$ where we stop the numerics ( $R$ must be chosen sufficiently large). Using the linearity of our ODE's, this minimizing condition leads to simple algebraic relations between the initial data and the values of $a_{1}(R), b_{1}(R)$ for a fundamental set of solutions, from which the initial values can be computed numerically. According to Figures 11 and 12, we already know qualitatively how the resulting $\left(a_{1}, b_{1}\right)$-plot is supposed to look like. This is helpful for checking the numerical results and for finding the best values for $R$. (More precisely, the best value for $R$ is, roughly speaking, the value where the $\left(a_{1}, b_{1}\right)$-plot 
starts to look like a closed curve. If $R$ is chosen much larger than this, the numerical inaccuracies pile up in a such way that our method of computing the initial data from $a_{1}(R)$ and $b_{1}(R)$ is no longer reliable.)

This procedure can be carried out for different values of $\kappa$, and gives the plots of Figures 13 to 18. For very small values of $\kappa$, the plots look like those in Figure 13, and one sees that $\left(\hat{a}_{2}(r), \hat{b}_{2}(r)\right)$ does not tend to zero for large $r$. The shape of the $\left(\hat{a}_{2}, \hat{b}_{2}\right)$-plot does not change over many magnitudes in $\kappa$ (see Figures 12, 14, 15), showing too that our numerics are well-behaved. For $\kappa \approx 0.02$, the form of the plots changes drastically (see Figures 16, 17, 18). One sees that $\left(\hat{a}_{2}, \hat{b}_{2}\right)$ still do not go to the origin and that it becomes impossible to satisfy even condition (8.22). If $\kappa$ is further increased, both $\left(a_{1}, b_{1}\right)$ and $\left(\hat{a}_{2}, \hat{b}_{2}\right)$ go to infinity for large $r$, no matter how the initial data is chosen. From this, we conclude that the ground state solution of Figure 11 is linearly stable.

Our method also applies to the excited states. For the first excited state and the lowest negative-mass state (i.e., for the solutions of the Figures 2 and 5), the solutions of the linearized equations for small $\kappa$ are plotted in Figures 19 and 20. The $\left(a_{2}, b_{2}\right)$-curves are again of similar shape as the corresponding $\alpha-\beta$-plot; the $\left(a_{1}, b_{1}\right)$-curve resembles the $\alpha-\beta$-plot for the next higher excited state (i.e., in Figure 19 for the second excited state, and in Figure 20 for the first excited negative-mass state). It is again useful to introduce the variables $\hat{a}_{2}, \hat{b}_{2}$. A numerical analysis of the equations for different values of $\kappa$ shows that these solutions are also stable.

It might seem a bit surprising that even the excited states are linearly stable. Actually, this can already be understood qualitatively from Figure 19 and the following heuristic argument. Thus, if the solution (for small coupling) were unstable, then the solution $\left(a_{j}, b_{j}, A_{1}, T_{1}\right)$ of the linearized equations (8.8)-8.13) would, to first order, describe the decay of the bound state. Therefore the $\left(a_{j}, b_{j}\right)$-plots give us information into which state the wave functions tend to decay. The $\left(a_{2}, b_{2}\right)$-plot is not interesting in this respect, because it looks like the $\alpha-\beta$-plot and only yields information about a time-dependent phase transformation of the wave functions. The $\left(a_{1}, b_{1}\right)$-plot, however, is helpful. According to Figure 19, the wave function tends to decay into the second excited state. This is surprising; one might instead have expected the tendency to decay into the ground state. Since the energy of the second excited state is higher than that of the first excited state, it would seem physically reasonable that this decay cannot happen spontaneously; this gives a simple explanation for stability.

We point out that these stability results are only valid for weak coupling, i.e., for small (scaled) mass $m$. If $m$ comes into the region where the mass spectrum of Figure 7 starts to have the form of a spiral curve, the numerical behavior of the linearized equations becomes much more difficult to analyze and does no longer allow simple conclusions. It is thus very helpful to study the stability in this regime via a different method, which we will now describe.

The existence of the spiral curve (c.f. Figure 21), enables us to obtain information regarding the stability properties of these solutions, using Conley Index theory (see [14, Part IV]). Indeed, as the figure shows, if $m>m_{1}$, there are no solutions while at $m=m_{1}$, the solution $P_{1}$ suddenly appears. This solution is "degenerate", and has Conley index $\overline{0}$ (the homotopy type of a one-point, pointed space). For $m<m_{1}$, the solution $P_{1}$ bifurcates into two solutions $Q_{1}$ and $Q_{2}$. The solution $Q_{1}$, being a "continuation" of the stable solution $Q_{0}$ ( $m$ is near 0$)$, must also be stable; this follows from Conley's Continuation Theorem [14, Thm. 23.31]. In fact, since $Q_{0}$ being stable implies that the Conley index of $Q_{0}$ is $\Sigma^{0}$, (the pointed zero sphere), the continuation theorem implies that 
the Conley index of $Q_{1}$ must also be $\Sigma^{0}$, and thus $Q_{1}$ is also a stable solution. (Moreover, the same argument shows that all points on the curve between 0 and $P_{1}$ correspond to stable solutions; this can also be checked numerically). Since the Conley indices of $Q_{1}$ and $Q_{2}$ must "cancel" (i.e., the index of $Q_{1}$ and $Q_{2}$ taken together must be $\overline{0}$ ), this implies that $Q_{2}$ cannot be stable, and in fact, the index of $Q_{2}$ is $\Sigma^{1}$, the pointed 1-sphere. (In fact, all points on the curve between $P_{1}$ and $P_{2}$ correspond to unstable solutions; one can also check this numerically.) Similar reasoning can be applied to solutions near $P_{2}, P_{3}$, $\ldots$, and so on. (We remark that only $m$, and not $\omega$, can serve as a bifurcation parameter; we show this in Appendix B.)

These general Conley Index techniques also enable us to show that for each $n$, these spiral curves must tend, as $\alpha_{1} \rightarrow \infty$, to a limiting configuration $\Gamma_{n}$, which is either a single point, or is a "limit cycle" $S^{1}$; i.e. a topological circle (we assume, as the numerics indicate, that the curve "spirals inwards"). In fact, were this not the case, then for each value of $m$ between 0 and $m_{1}$, the corresponding solution set would form an "isolated invariant set" (c.f. 14]), and so, again by Conley's Continuation Theorem, their Conley indices would all be the same. However, for $m$ near 0 , the index of the isolated invariant set is $\Sigma^{0}$, while for $m$ near $m_{1}$, the corresponding isolated invariant set has index $\overline{0}$. Since these two indices are different, we have a contradiction.

It follows from this last result that for a point $(\hat{m}, \hat{m}-\hat{\omega})$ on $\Gamma_{n}$, there are an infinite number of solutions with $m=\hat{m}$, as well as an infinite number of solutions with $m-\omega=$ $\hat{m}-\hat{\omega}$. For parameter points not meeting $\Gamma_{n}$, there are at most a finite number of solutions.

\section{A Appendix: Justification of the Ansatz for the Spinor De- pendence}

In this section, we consider the general form of the spinors in static, spherically symmetric systems and derive the Einstein-Dirac equations for these systems. This analysis will clarify the ansatz' (3.4) and (3.10) for the wave functions, which was made in Section 3 without a detailed explanation.

The Dirac wave functions $\Psi_{1}, \Psi_{2}$ of a general two-fermion system can be written in the form

$$
\Psi_{a}(\vec{x}, t)=A(\vec{x}, t) e_{a} \quad, \quad a=1,2 \quad .
$$

where $A_{a}^{\alpha}=\Psi_{a}^{\alpha}$ is a $(4 \times 2)$-matrix and where $\left(e_{a}\right)$ again denotes the standard basis of the two-component Pauli spinors. The system being static means that the time dependence of $A$ has the form of a plane wave,

$$
A(\vec{x}, t)=e^{-i \omega t} A(\vec{x})
$$

The simplest way to characterize the spherical symmetry of the spinors is to demand that the angular dependence of $A$ is described only by the submatrices $\mathbb{1}$ and $\sigma^{r}$; i.e.

$$
A(\vec{x})=\left(\begin{array}{l}
v_{1}(r) \mathbb{1}+v_{2}(r) \sigma^{r} \\
v_{3}(r) \mathbb{1}+v_{4}(r) \sigma^{r}
\end{array}\right)
$$

with complex functions $v_{1}, \ldots, v_{4}$. This form of $A$ can be derived if one requires that the total angular momentum is zero and that all the expectation values $\langle\mathcal{O}\rangle=\sum_{a=1}^{2} \overline{\Psi_{a}} \mathcal{O} \Psi_{a}$ of the spin matrices are spherically symmetric. In a simplified argument, this form can be 
understood directly from the fact that the presence of any matrices $\sigma^{\vartheta}, \sigma^{\varphi}$ would destroy the radial symmetry in (A.2).

The ansatz (A.1), A.2) for the wave functions is a linear combination of (3.4) and (3.10). From this, we immediately obtain the corresponding Dirac equations. Namely, the complex two-spinors $\Phi=\left(\Phi_{1}, \Phi_{2}\right)$ and $\Xi=\left(\Xi_{1}, \Xi_{2}\right)$ with

$$
\begin{array}{ll}
\Phi_{1}=r T^{-\frac{1}{2}} v_{1}, & \Xi_{1}=r T^{-\frac{1}{2}} v_{2} \\
\Phi_{2}=-i r T^{-\frac{1}{2}} v_{4}, & \Xi_{2}=-i r T^{-\frac{1}{2}} v_{3}
\end{array}
$$

satisfy, (in analogy to $(3.9)$ ), the equations

$$
\begin{aligned}
& \sqrt{A} \Phi^{\prime}=\left[\omega T\left(\begin{array}{cc}
0 & -1 \\
1 & 0
\end{array}\right)+\frac{1}{r}\left(\begin{array}{cc}
1 & 0 \\
0 & -1
\end{array}\right)-m\left(\begin{array}{ll}
0 & 1 \\
1 & 0
\end{array}\right)\right] \Phi \\
& \sqrt{A} \Xi^{\prime}=\left[\omega T\left(\begin{array}{cc}
0 & -1 \\
1 & 0
\end{array}\right)-\frac{1}{r}\left(\begin{array}{cc}
1 & 0 \\
0 & -1
\end{array}\right)-m\left(\begin{array}{ll}
0 & 1 \\
1 & 0
\end{array}\right)\right] \Xi
\end{aligned}
$$

In Section 3, we argued that the reality of the coefficients in (3.9) allows us to choose real spinors. This procedure simplified the Dirac equations considerably, but it might be too restrictive to describe the general solution of the Einstein-Dirac equations. In order to analyze the situation more carefully, we first note that the function

$$
F(r):=\Phi(r)^{*}\left(\begin{array}{cc}
0 & -i \\
i & 0
\end{array}\right) \Phi(r)
$$

is independent of $r$, as is obvious from (A.3). The boundary conditions at the origin, $\Phi_{1}(0)=0=\Phi_{2}(0)$, imply that $F$ must vanish identically, and thus the product $\overline{\Phi_{1}} \Phi_{2}$ is real. This means that $\Phi_{1}$ and $\Phi_{2}$ must be real up to a common phase factor, i.e.

$$
\Phi_{1}(r)=e^{i \alpha} f_{1}(r), \quad \Phi_{2}(r)=e^{i \alpha} f_{2}(r)
$$

with real functions $f_{1}, f_{2}$. Again as a consequence of the Dirac equation (A.3), the phase $\alpha$ is independent of $r$. A similar argument applies to $\Xi$ and gives

$$
\Xi_{1}=e^{i \beta} g_{1}(r), \quad \Xi_{2}=e^{i \beta} g_{2}(r)
$$

with real functions $g_{1}, g_{2}$ and $\beta \in \mathbb{R}$.

We shall now compute the energy-momentum tensor. First of all, the spherical symmetry of the spinors implies that the off-diagonal components $T_{\vartheta}^{t}, T_{\varphi}^{t}, T_{\vartheta}^{r}, T_{\varphi}^{r}, T_{\varphi}^{\vartheta}$ vanish and that $T_{\vartheta}^{\vartheta}=T_{\varphi}^{\varphi}$. Thus we must only consider $T_{r}^{t}$ and the diagonal components $T_{t}^{t}, T_{r}^{r}$, $T_{\vartheta}^{\vartheta}$ of the energy-momentum tensor. As a first step, we verify that the contribution (4.3) of the variation of $B$ vanishes: According to (2.20), the trace in (4.3) is zero if $\delta g_{m k}$ is diagonal. Thus we must only consider $T_{r}^{t}$, and we may therefore assume that the indices $m, k$ are either $m=t, k=r$ or $m=r, k=t$. Furthermore, the spherical symmetry (A.2) implies that the expectation of the pseudovector $\gamma^{5} G^{p}$ only has a component in the time and radial directions,

$$
\sum_{a=1}^{2} \overline{\Psi_{a}} \gamma^{5} G^{p} \Psi_{a}=0 \quad \text { for } p=\varphi \text { or } p=\vartheta
$$


We conclude that both indices $m$ and $p$ in (4.3) must be equal to either $r$ or $t$, and the antisymmetry of the $\epsilon$-tensor implies that the remaining indices $j, n$ must coincide either with $\vartheta$ or $\varphi$. Thus we must only consider the trace in (4.3) for the combination

$$
\operatorname{Tr}\left(G^{k}\left(\partial_{\vartheta} G_{\varphi}-\partial_{\varphi} G_{\vartheta}\right)\right) \quad .
$$

But, according to (2.16), (2.17) and (2.11), we have $\partial_{\vartheta} G_{\varphi}=\partial_{\varphi} G_{\vartheta}$, so that (A.7) vanishes.

We conclude that the energy-momentum tensor is again given by (4.4). In order to compute the trace in (4.4), it is useful to first notice that, if we write the Dirac matrices as $(2 \times 2)$ block matrices, then $G^{t}$ is diagonal with entries proportional to the identity, whereas $G^{r}$ is off-diagonal with submatrices which are multiples of $\sigma^{r}$. This implies that the mixed contribution (i.e., the contribution proportional to $\bar{\Phi} \Xi$ or $\bar{\Xi} \Phi$ ) to $T_{t}^{t}$ and $T_{r}^{r}$ vanish. Using the explicit form of $G^{\vartheta}$ together with (3.1), we conclude that the mixed contribution also vanishes in $T_{\vartheta}^{\vartheta}$. Thus the energy-momentum tensor of the system is simply the sum of the energy-momentum tensors corresponding to the spinors $\Phi$ and $\Xi$. As a consequence, the constant phase factors in (A.5), (A.6) are irrelevant; we can without loss of generality assume that $\Phi$ and $\Xi$ are real. Using (4.5), we end up with the formulas

$$
\begin{aligned}
& T_{j}^{i}= T_{j}^{i}[\Phi]+T_{j}^{i}[\Xi] \quad \text { with } \\
& T_{j}^{i}[\Phi]= r^{-2} \operatorname{diag}\left(2 \omega T^{2}|\Phi|^{2},-2 \omega T^{2}|\Phi|^{2}+4 T r^{-1} \Phi_{1} \Phi_{2}+2 m T\left(\Phi_{1}^{2}-\Phi_{2}^{2}\right),\right. \\
&\left.-2 T r^{-1} \Phi_{1} \Phi_{2},-2 T r^{-1} \Phi_{1} \Phi_{2}\right) \\
& T_{j}^{i}[\Xi]= r^{-2} \operatorname{diag}\left(2 \omega T^{2}|\Xi|^{2},-2 \omega T^{2}|\Xi|^{2}-4 T r^{-1} \Xi_{1} \Xi_{2}+2 m T\left(\Xi_{1}^{2}-\Xi_{2}^{2}\right),\right. \\
&\left.2 T r^{-1} \Xi_{1} \Xi_{2}, 2 T r^{-1} \Xi_{1} \Xi_{2}\right)
\end{aligned}
$$

Thus the Einstein-Dirac equations take the form (A.3), A.4 and

$$
G_{j}^{i}=-8 \pi\left(T_{j}^{i}[\Phi]+T_{j}^{i}[\Xi]\right)
$$

with $T_{j}^{i}$ given by (A.10), (A.11). This is a generalization of the system (5.4)- (5.7). It is quite remarkable that the energy-momentum tensor is just the sum of $T_{j}^{i}[\Phi]$ and $T_{j}^{i}[\Xi]$. Similar to (5.8), the normalization condition for the wave functions takes the form

$$
\int_{0}^{\infty}\left(|\Phi|^{2}+|\Xi|^{2}\right) \frac{T}{\sqrt{A}} d r=\frac{1}{4 \pi}
$$

We now qualitatively describe how the solutions of this generalized system can be constructed and how we recover the solutions of Section 7 . The scaling technique of Section 6 can again be applied and consequently we can omit the conditions $T(\infty)=1$ and (A.12) if we instead set $T(0)=1=m$. Then the solutions are characterized by the three parameters $\omega, \Phi_{1}^{\prime}(0), \Xi_{2}^{\prime}(0)$ (notice that a Taylor expansion around $r=0$ yields, in analogy to (5.10), the constraints $\left.\Phi_{2}^{\prime}(0)=0=\Xi_{1}^{\prime}(0)\right)$. Compared to the situation in Section 0, we thus have one additional continuous parameter to describe the solution. At first sight, this might seem to imply that we can now construct, for given $\omega$, a continuous one-parameter family of solutions. Then our ansatz' (3.4) and (3.10) would just correspond to two special points of this continuum of solutions, and it would become unsatisfying that we just picked these two special solutions for the discussion of the mass spectrum and the 
stability. However, the additional free parameter is illusory due to the fact that we also have one additional constraint at infinity. Namely, we must (for given $\omega$ ) adjust $\Phi_{1}^{\prime}(0)$ and $\Xi_{2}^{\prime}(0)$ in such a way that both the $\left(\Phi_{1}, \Phi_{2}\right)$ and the $\left(\Xi_{1}, \Xi_{2}\right)$ curves tend to zero for large $r$. For generic $\omega$, these conditions will only be satisfied for a discrete set of initial values $\left(\Phi_{1}^{\prime}(0), \Xi_{2}^{\prime}(0)\right)$. The choices $\left(\Phi_{1}^{\prime}(0)=0, \Xi_{2}^{\prime}(0)\right)$ and $\left(\Phi_{1}^{\prime}(0), \Xi_{2}^{\prime}(0)=0\right)$ are both allowable; they correspond to the solutions constructed in Section 7. After scaling, this shows that for generic $m$, the Einstein-Dirac equations only admit a discrete number of solutions.

We note that these considerations do not rule out the possibility that the general ansatz for the spinors might lead to some additional solutions. We did not study the general equations systematically and can only say qualitatively that it seems difficult to arrange that there are simultaneous normalizable solutions of (A.3) and (A.4). The existence of solutions of this type, however, remains an open question.

\section{B Appendix: Justification of Using $m$ as the Bifurcation Parameter}

We show in this section, first that $\omega$ is unsuitable as a bifurcation parameter, and second that $m$ can serve as a bifurcation parameter. In Conley Index theory, a parameter can only be used as a bifurcation parameter if it remains well-defined and fixed when perturbations of the solutions are considered. The basic reason why $m$ and $\omega$ must be treated differently can already be understood from the general form of the Dirac equation in (1.1). The mass $m$ enters as an a-priori given parameter into the Dirac equation, whereas the energy $\omega$ of the fermion is only determined by the solution $\Psi$. This means that if we consider a variation of a solution, $m$ can be considered as a fixed parameter, while $\omega$ will in general change. If the perturbation of the solution is not static, the energy of the fermion will in general become time-dependent, and $\omega$ will no longer be a well-defined parameter.

Although being correct in principle, this argument is too simple and not fully convincing. First of all, the situation becomes more complicated by our scaling technique, which also changes $m$ and thus makes it impossible to consider the mass as a fixed parameter throughout. Furthermore, $\omega$ is uniquely determined by the solutions $(\alpha, \beta, A, T)$ of (5.4)-(5.7). It enters as a parameter into the linearized equations in a similar way as $m$ does, and it is not obvious from these equations why the two parameters $m$ and $\omega$ should play such different roles for stability considerations. Therefore we will show in detail that solutions of the linearized equations do not determine $\omega$, whereas $m$ is still a well-defined parameter.

In order to show that $\omega$ is not well-defined, we generalize the ansatz of Section 8 in the way that we also allow $\omega$ to be time-dependent. In analogy to (8.2)-(8.5) and (8.6), 8.7), we consider a perturbation of $\omega$ of the form

$$
\omega(t)=\omega+\epsilon \omega_{1} e^{\kappa t} \quad .
$$

Since $\omega$ represents a frequency, i.e. the time-derivative of a phase, the correct generalization of equation (8.1) is to replace the phase factor $e^{-i \omega t}$ by

$$
\exp \left(-i \int_{0}^{t} \omega(s) d s\right)
$$

The ansatz for $a_{j}, b_{j}, A_{1}, T_{1}$ then remains the same as before, given by (8.2)-(8.5) and 
(8.6), (8.7). Thus the spinors $z_{1}, z_{2}$ are given by

$$
\begin{aligned}
& z_{1}(r, t)=e^{-i \int_{0}^{t} \omega(s) d s}\left[\alpha(r)+\varepsilon\left(a_{1}(r, t)+i a_{2}(r, t)\right]\right. \\
& z_{2}(r, t)=e^{-i \int_{0}^{t} \omega(s) d s}\left[\beta(r)+\varepsilon\left(b_{1}(r, t)+i b_{2}(r, t)\right]\right.
\end{aligned}
$$

Into these equations, we substitute (B.1) and consider only the first-order terms in $\varepsilon$. This gives

$$
\begin{aligned}
z_{1}(r, t) & =e^{-i \omega t}\left(1-i \varepsilon \int_{0}^{t} \omega_{1} e^{\kappa s} d s\right)\left[\alpha(r)+\varepsilon\left(a_{1}(r, t)+i a_{2}(r, t)\right]\right. \\
& =e^{-i \omega t}\left[\alpha(r)-i \varepsilon \frac{\omega_{1}}{\kappa}\left(e^{\kappa t}-1\right) \alpha(r)+\varepsilon\left(a_{1}(r, t)+i a_{2}(r, t)\right]\right. \\
& =e^{-i \omega t}\left[\left(1+i \varepsilon \frac{\omega_{1}}{\kappa}\right) \alpha(r)+\varepsilon\left(a_{1}(r, t)+i a_{2}(r, t)-i \varepsilon \frac{\omega_{1}}{\kappa} e^{\kappa t} \alpha(r)\right]\right. \\
& =e^{-i\left(\omega t-\varepsilon \frac{\omega_{1}}{\kappa}\right)}\left[\alpha(r)+\varepsilon\left(a_{1}(r, t)+i a_{2}(r, t)-i \varepsilon \frac{\omega_{1}}{\kappa} e^{\kappa t} \alpha(r)\right]\right.
\end{aligned}
$$

with a similar expression for $z_{2}(r, t)$. This looks quite similar to the original ansatz (8.1) and (8.2)-(8.5) except for two differences; namely, there is here an additional constant phase factor $\exp \left(i \varepsilon \frac{\omega_{1}}{\kappa}\right)$, together with the term

$$
-i \varepsilon \frac{\omega_{1}}{\kappa} e^{\kappa t} \alpha(r)
$$

The phase factor $\exp \left(i \varepsilon \frac{\omega_{1}}{\kappa}\right)$ plays no role in our analysis, since it falls out of all the equations (notice that the energy-momentum tensor (4.4) contains only terms of the form $\bar{\Psi} \cdots \Psi)$. What is interesting about the term (B.2), however, is that its time-dependence is again of the form $e^{\kappa t}$. It is thus consistent with our ansatz (8.6), and corresponds to the transformation

$$
a_{2}(r) \rightarrow a_{2}(r)-\varepsilon \frac{\omega_{1}}{\kappa} \alpha(r) \quad,
$$

and similarly

$$
b_{2}(r) \rightarrow b_{2}(r)-\varepsilon \frac{\omega_{1}}{\kappa} \beta(r) \quad .
$$

Thus the more general ansatz for $a_{j}, b_{j}, A_{1}, T_{1}$ whereby $\omega$ is replaced by (B.1) is the same as the original ansatz for these quantities if we transform $a_{2}$ and $b_{2}$ according to (B.3) and (B.4). Conversely, we may obtain an arbitrary time dependence in $\omega$, of the form (B.1), merely by transforming $a_{2}$ and $b_{2}$ according to (B.3) and (B.4). This means that a solution of the linearized equations only determines $\omega$ up to linear time-dependent perturbations of the form (B.1). Thus $\omega$ is not a well-defined parametert.

For the parameter $m$, however, the situation is completely differently. Namely if $\Psi$ is a solution of the time-dependent Dirac equation $G \Psi=m \Psi$, then we see from (2.7) that $(G \Psi \mid G \Psi)=m^{2}$. But as we noted earlier, (after (8.19)), this relation is also valid for the linearized equations. That is, $m$ is a well-defined parameter for the linearized equations.

\footnotetext{
${ }^{2}$ It is interesting to notice that the contribution to $\left(a_{2}, b_{2}\right)$ proportional to $(\alpha, \beta)$ which occurs in the transformation B.3), B.4 played an important role in our numerics. Namely, we saw in Section 8 that the $\left(a_{2}, b_{2}\right)$-plot looks very similar to the $(\alpha, \beta)$-plot (see Figure 11), which shows that this contribution is actually dominant for small $\kappa$. It caused problems in the numerics and forced us to introduce the new variables $\hat{a}_{2}, \hat{b}_{2}$ (see Figure 12). According to (B.3), B.4. , we can now understand the dominant contribution to $\left(a_{2}, b_{2}\right)$ in Figure 11 as describing a time-dependent perturbation of $\omega$ of the form (B.1).
} 

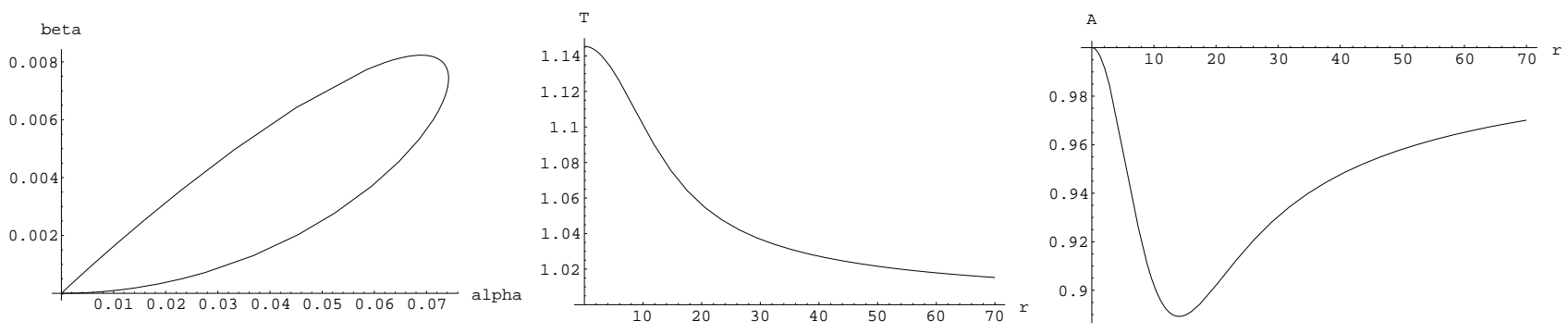

Figure 1: The ground state for $m=1, \alpha_{1}=0.02$; scaled parameter values: $m=0.5340$, $\omega=0.4994$
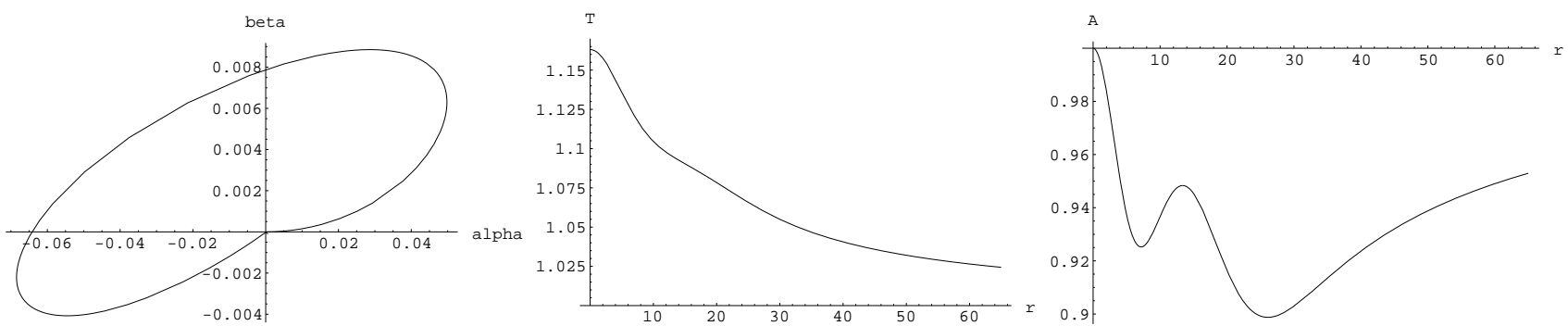

Figure 2: The first excited state for $m=1, \alpha_{1}=0.02$; scaled parameter values: $m=$ $0.7779, \omega=0.7326$
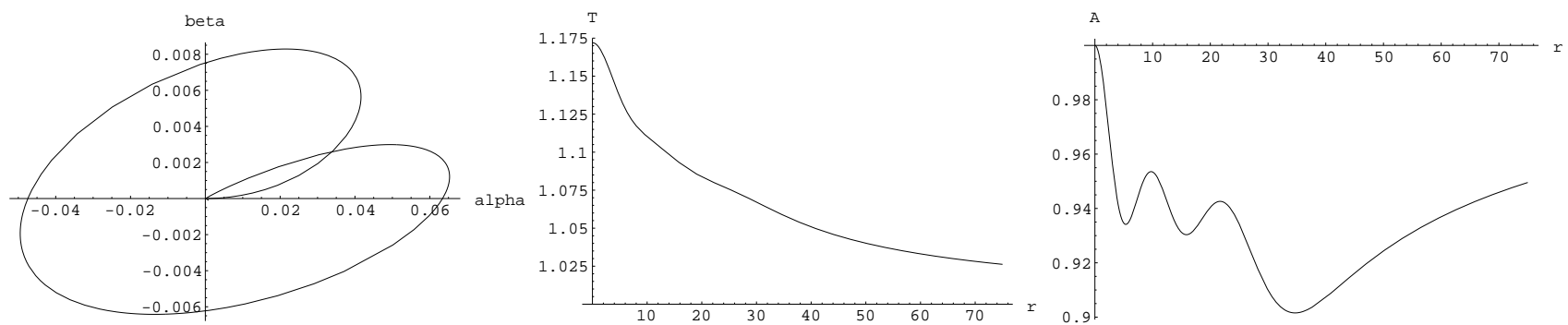

Figure 3: The second excited state for $m=1, \alpha_{1}=0.02$; scaled parameter values: $m=0.9616, \omega=0.9080$ 

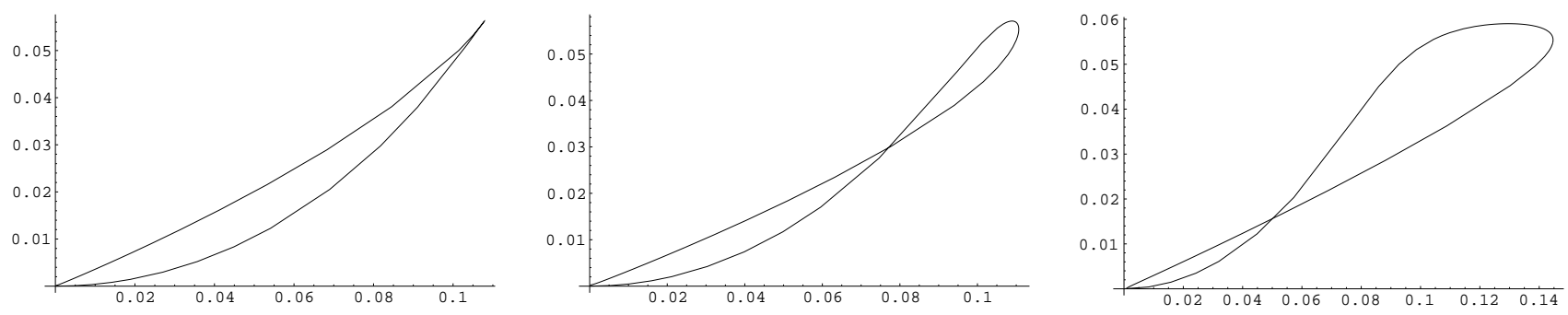

Figure 4: $\alpha$ - $\beta$-plot for the ground states at parameter values $m=1$ and $\alpha_{1}=$ $0.31,0.35,0.45$
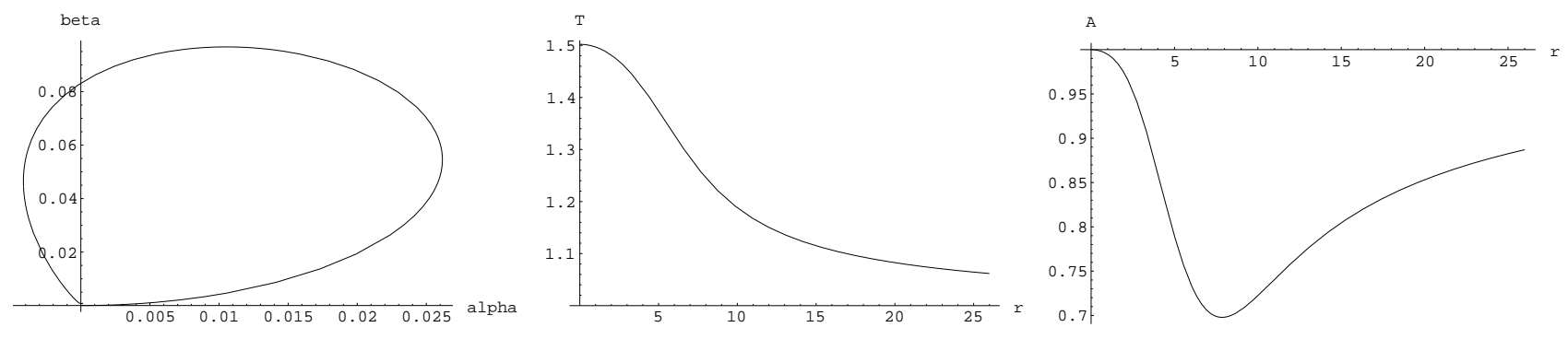

Figure 5: The lowest state for $m=-1, \alpha_{1}=0.02$; scaled parameter values: $m=-0.7567$, $\omega=0.6302$
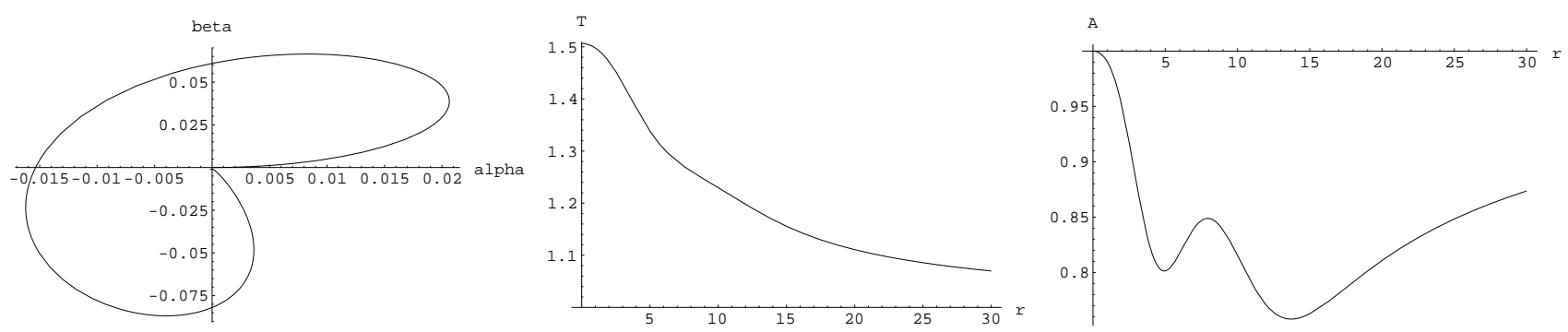

Figure 6: The next excited state for $m=-1, \alpha_{1}=0.02$; scaled parameter values: $m=$ $-0.9742, \omega=0.8391$ 


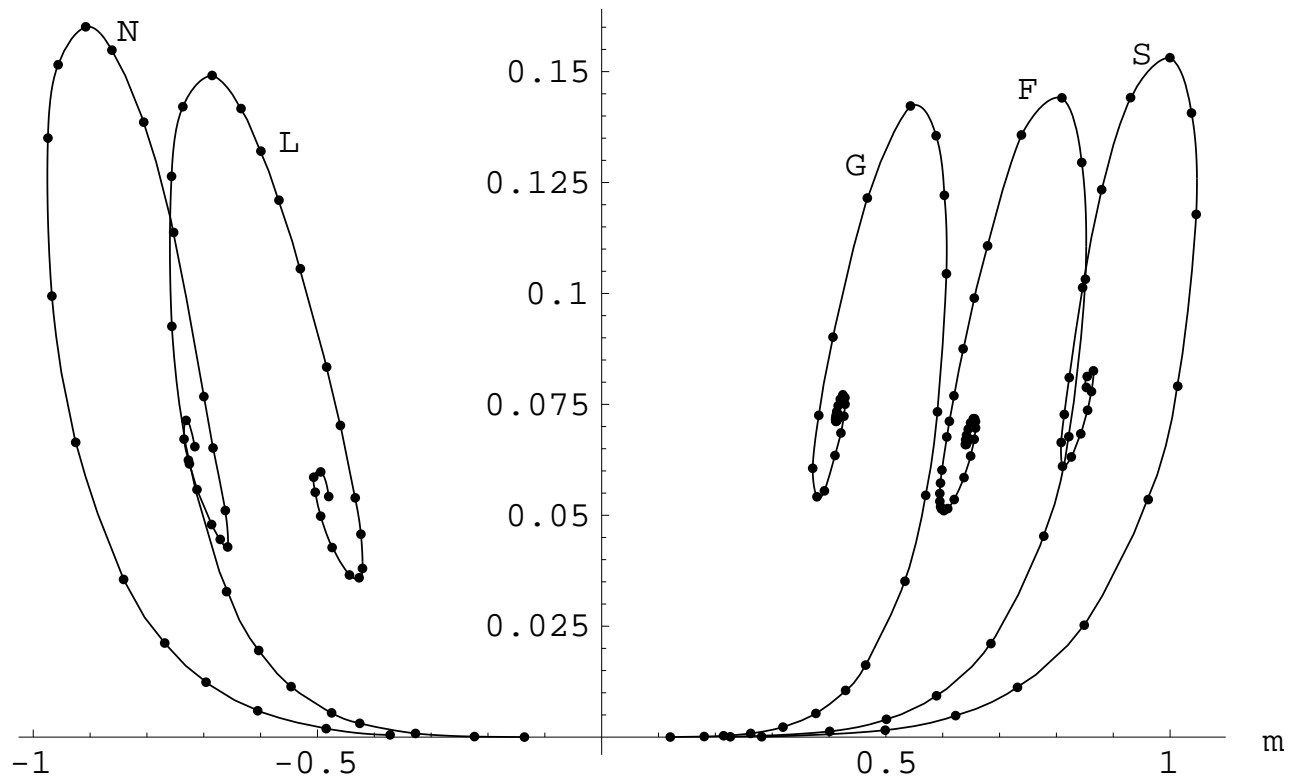

Figure 7: Binding Energy $|m|-\omega$ of the Fermions
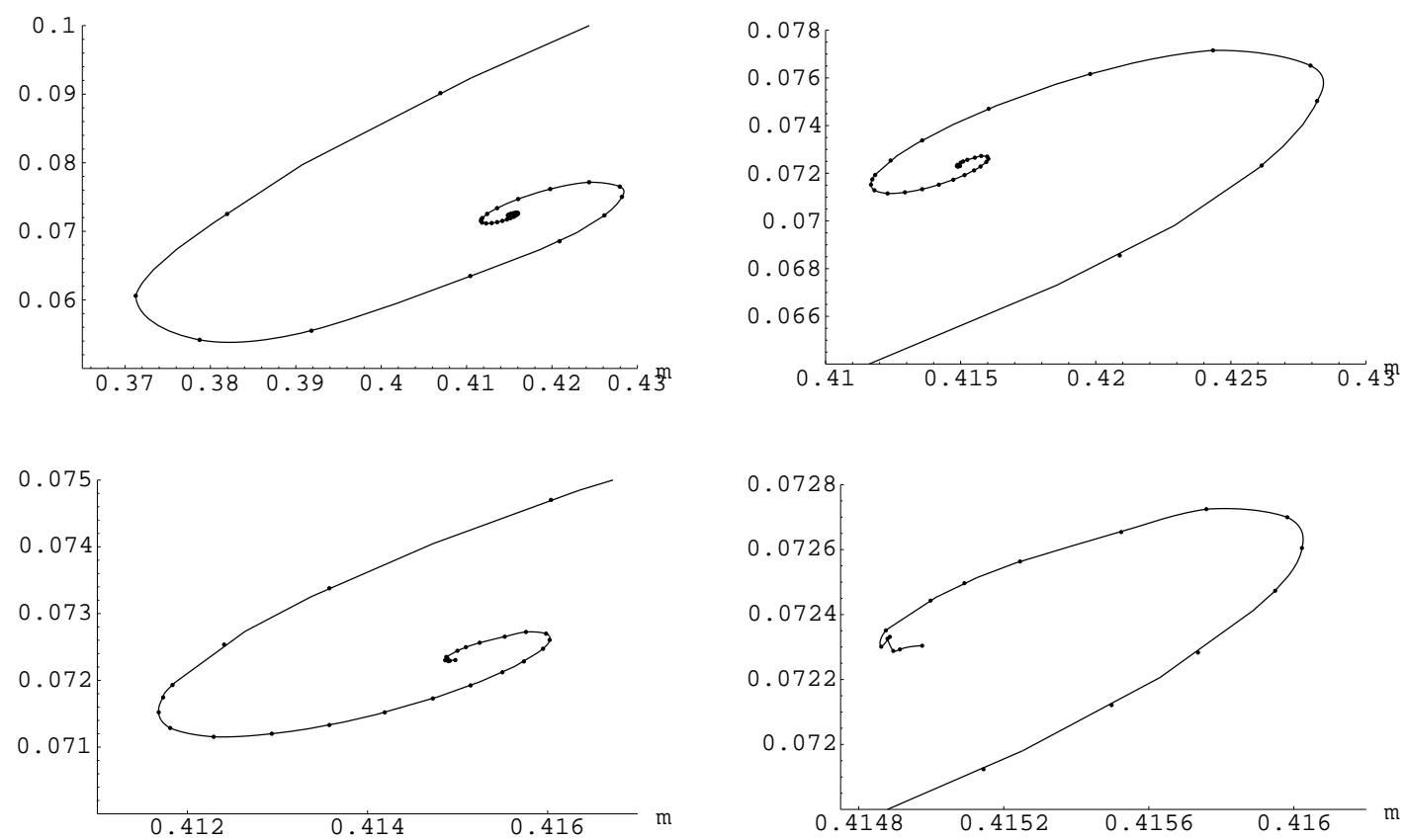

Figure 8: Binding Energy $|m|-\omega$ of the Ground State, Detailed Pictures 


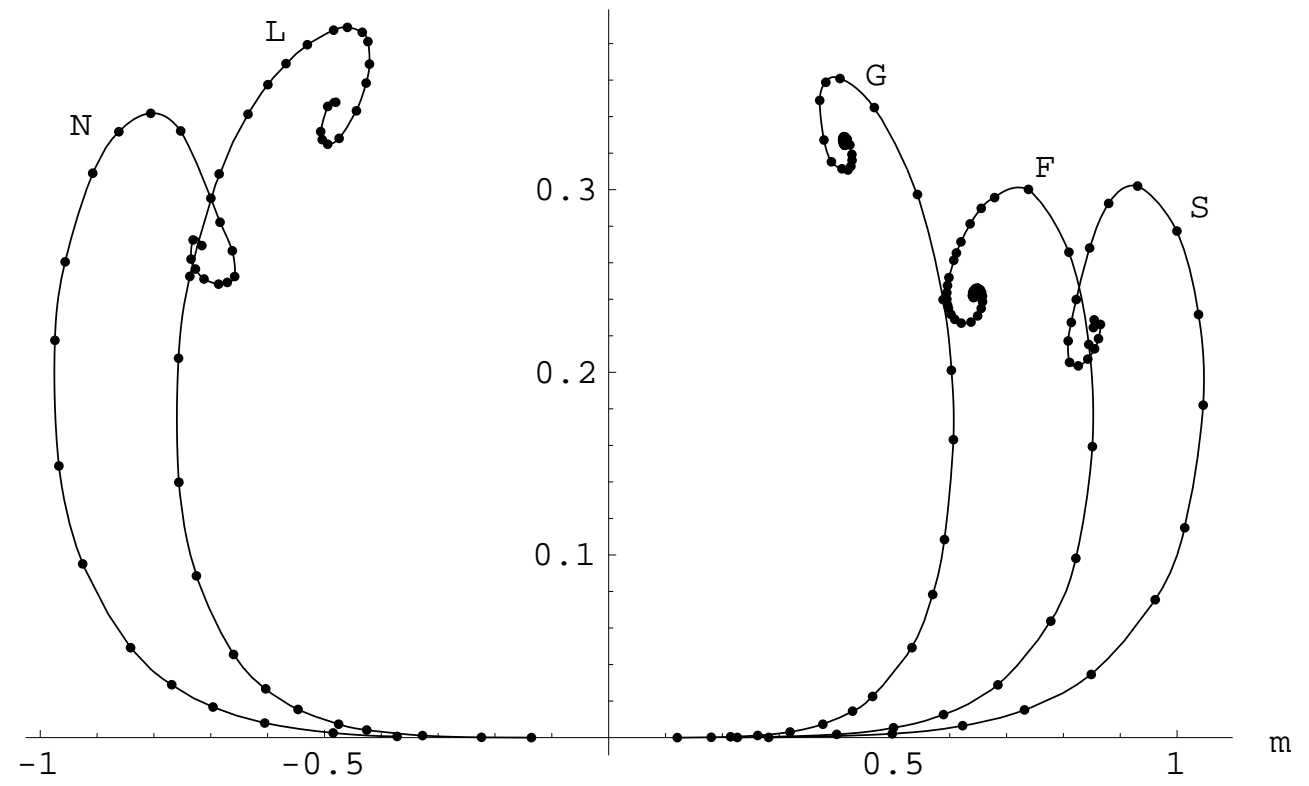

Figure 9: Total Energy $\rho-2 \omega$ of the Gravitational Field

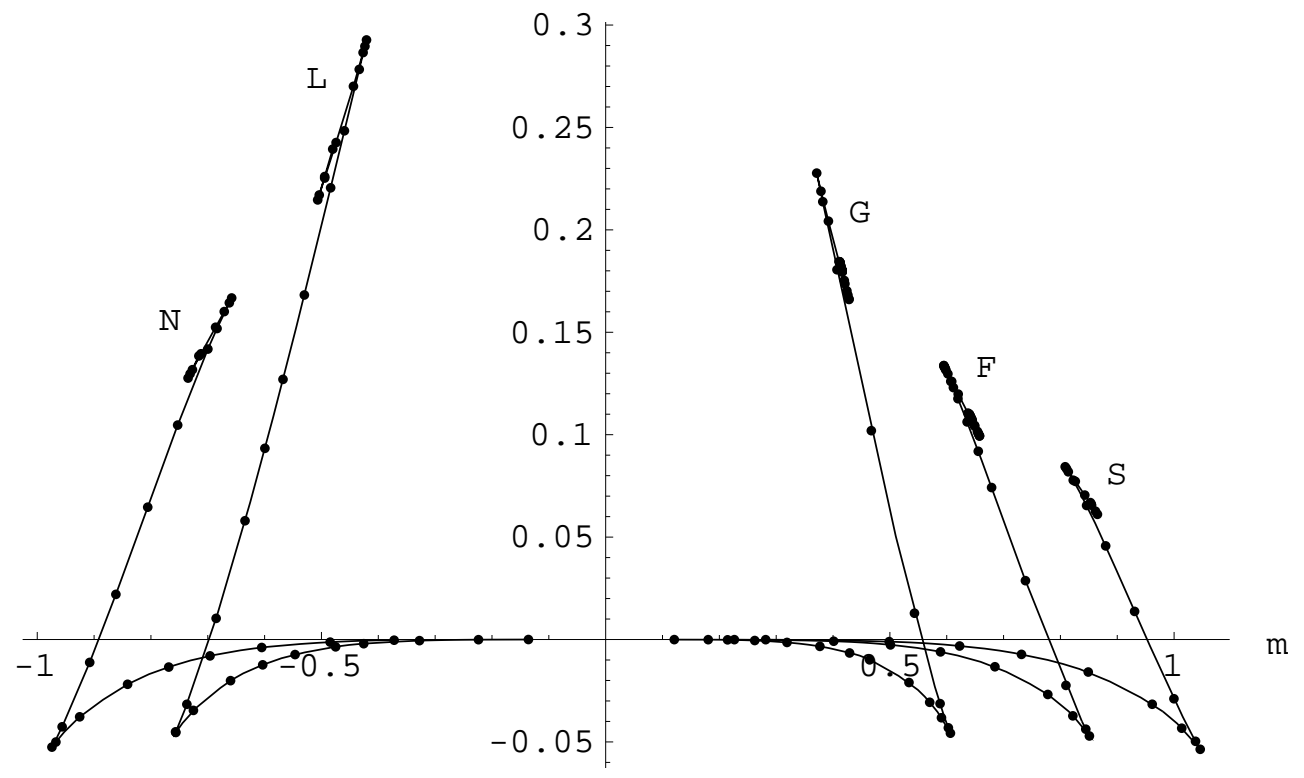

Figure 10: Total Binding Energy $\rho-2|m|$ 

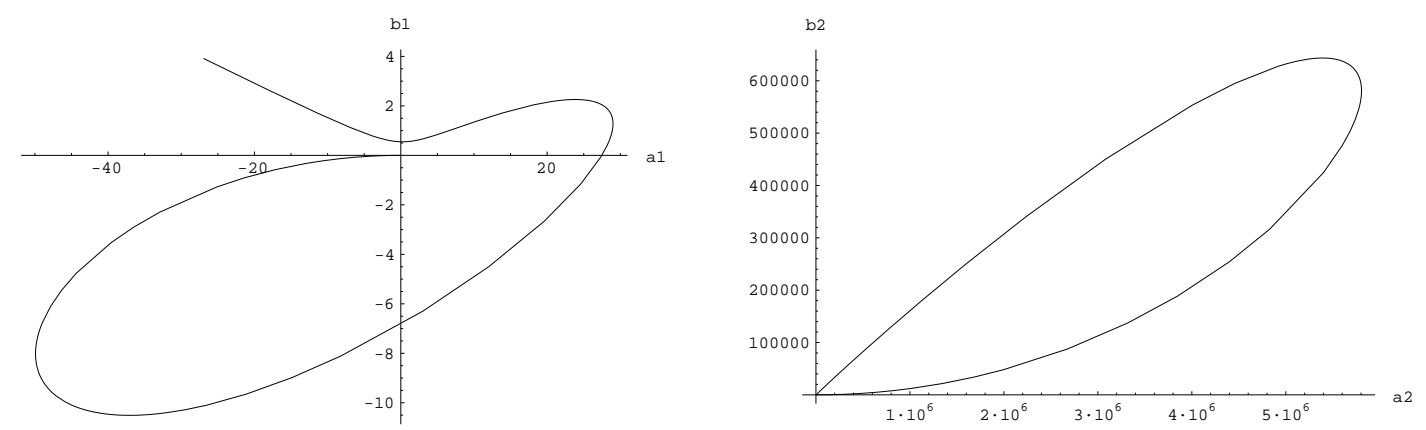

Figure 11: Perturbation of the ground state for $\kappa=10^{-6}$, variables $\left(a_{1}, b_{1}, a_{2}, b_{2}\right)$
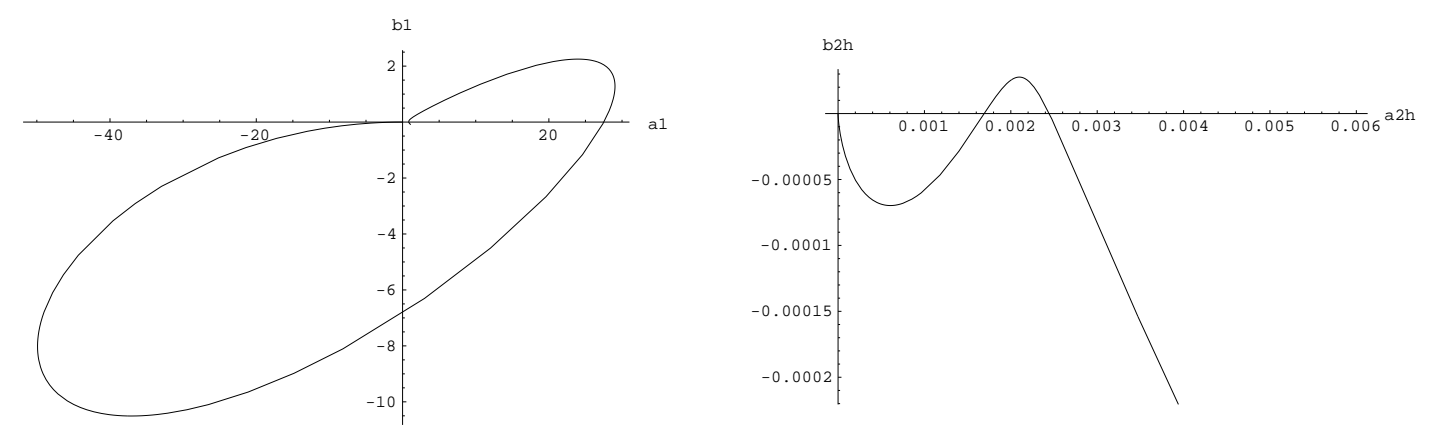

Figure 12: Perturbation of the ground state for $\kappa=10^{-6}$, variables $\left(a_{1}, b_{1}, \hat{a}_{2}, \hat{b}_{2}\right)$
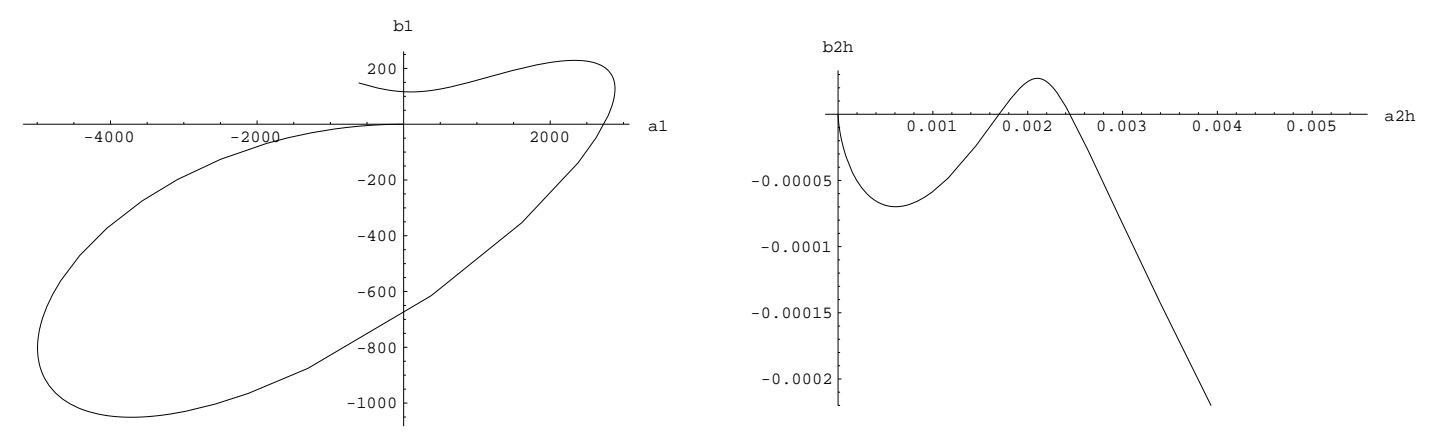

Figure 13: Perturbation of the ground state for $\kappa=10^{-8}$ 

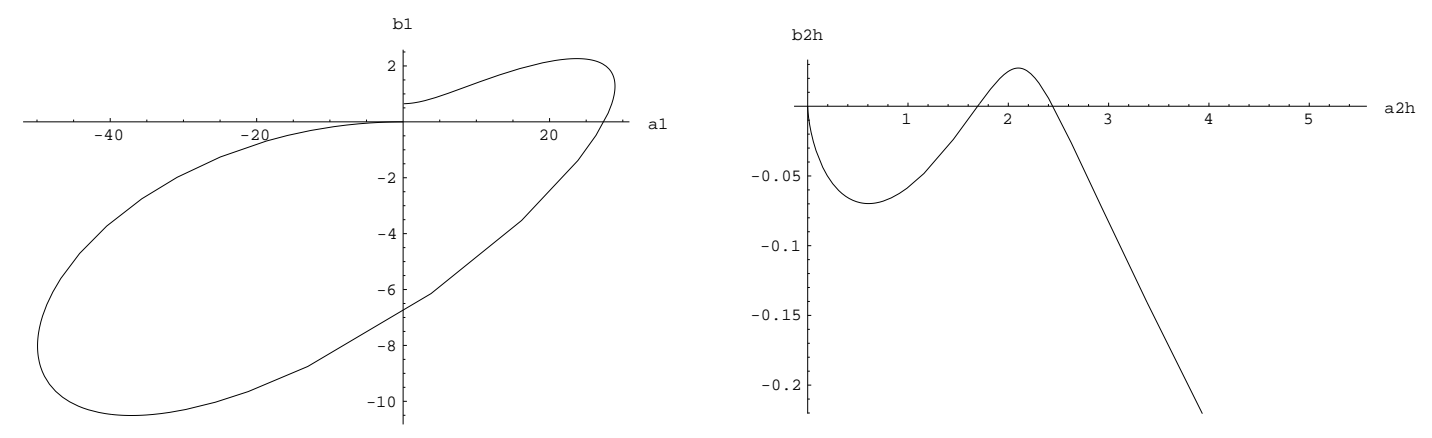

Figure 14: Perturbation of the ground state for $\kappa=10^{-3}$
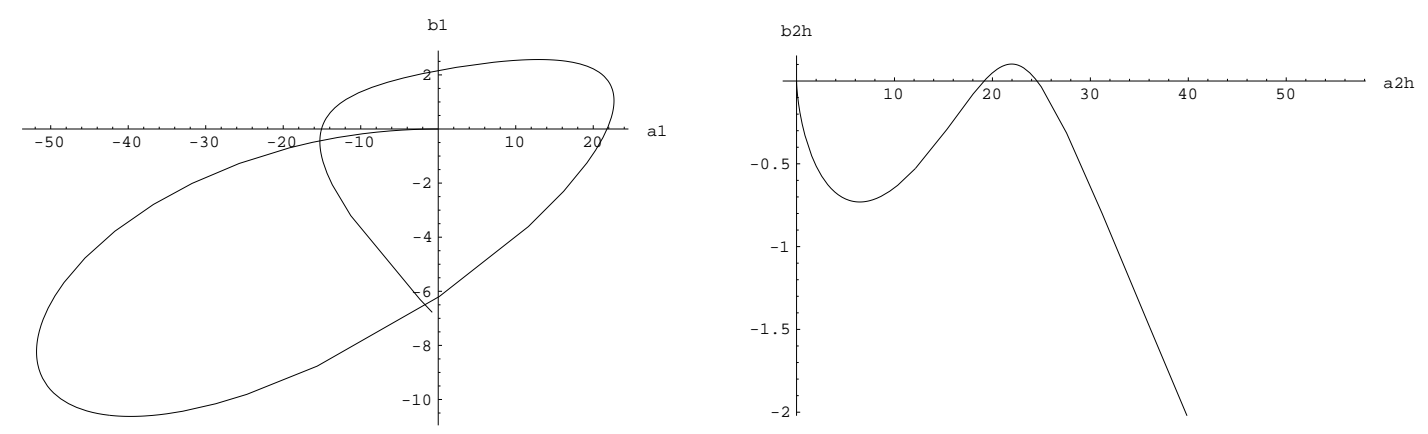

Figure 15: Perturbation of the ground state for $\kappa=0.01$
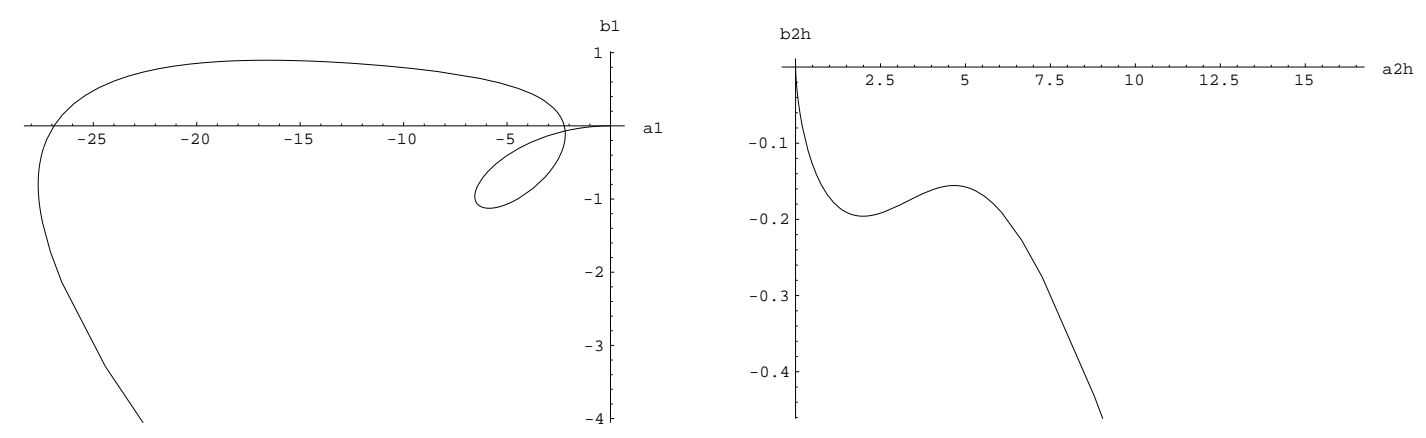

Figure 16: Perturbation of the ground state for $\kappa=0.02$
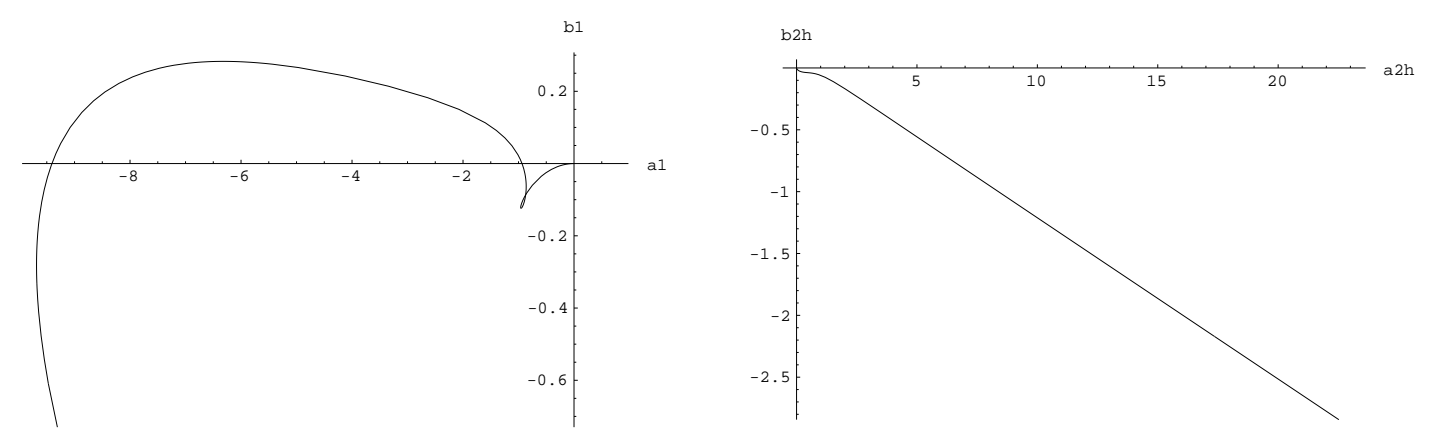

Figure 17: Perturbation of the ground state for $\kappa=0.023$ 

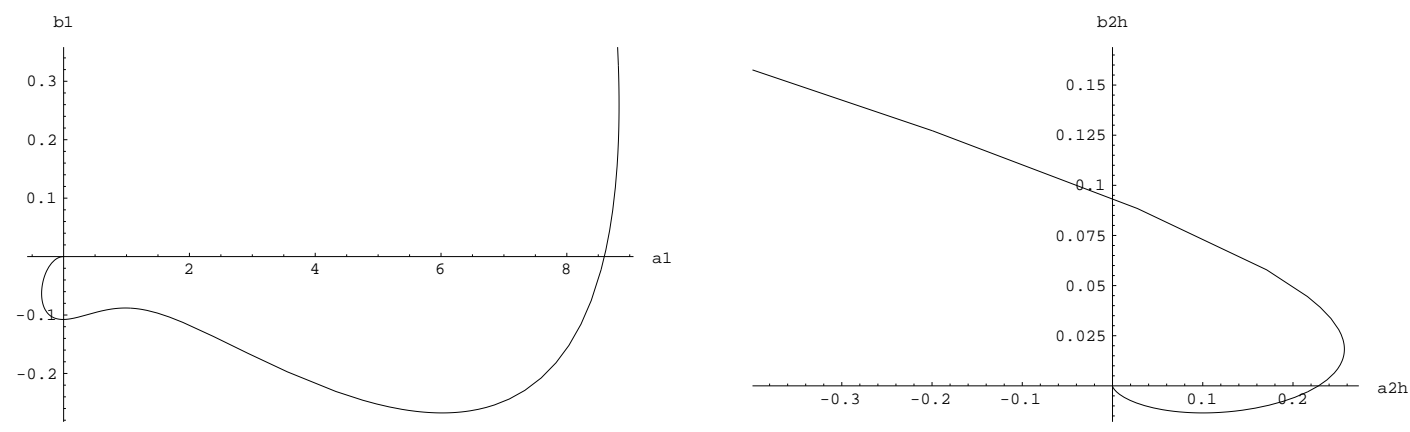

Figure 18: Perturbation of the ground state for $\kappa=0.03$
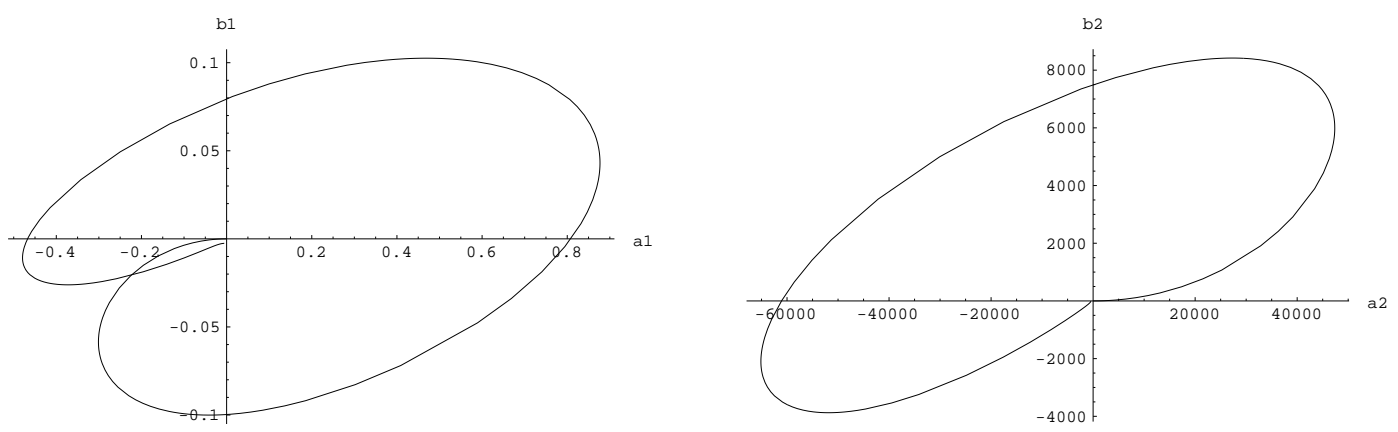

Figure 19: Perturbation of the first excited state for $\kappa=10^{-6}$
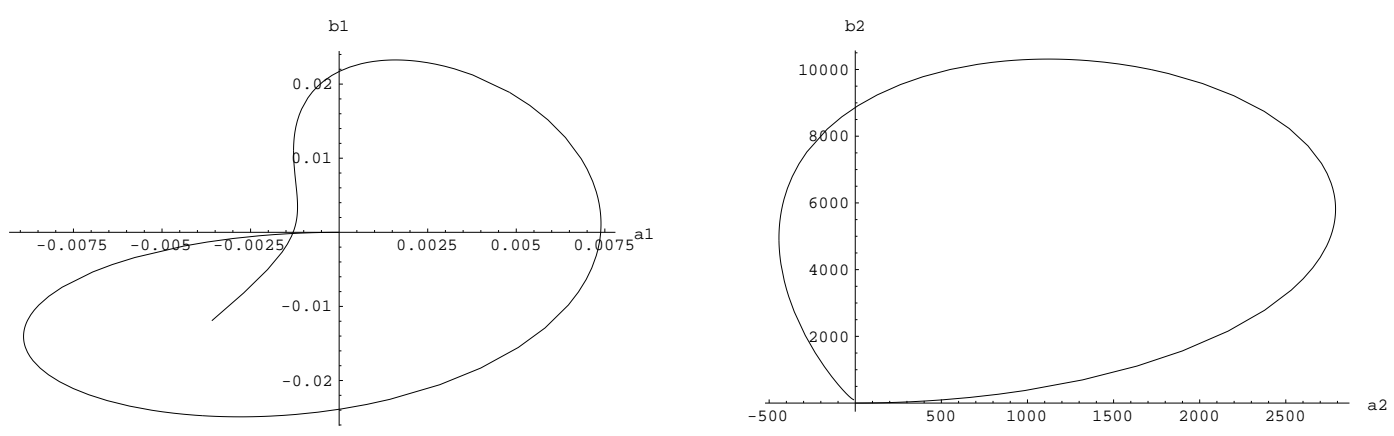

Figure 20: Perturbation of the lowest negative-mass state for $\kappa=10^{-6}$ 


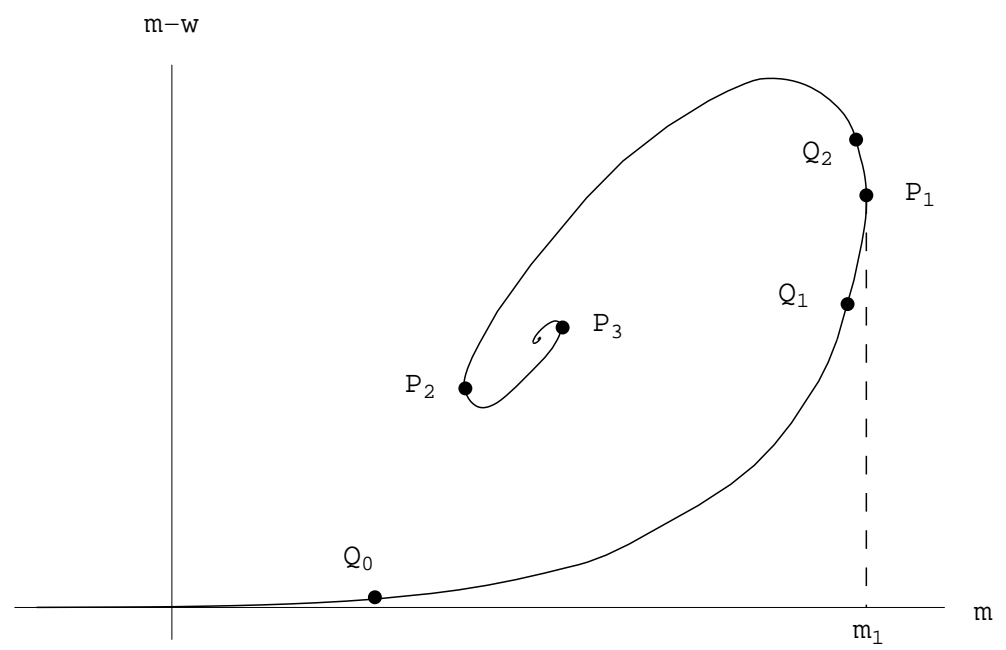

Figure 21: Mass-Energy spectrum for the $n^{\text {th }}$ excited state 


\section{References}

[1] Bartnik, R., and McKinnon, J., Phys. Rev. Lett. 61, 141-144, 1988

[2] Bizon, P., Phys. Rev. Lett. 64, 2844-847, 1990

[3] Breitenlohner, P., Forgacs, P. and Maison, D., Comm. Math. Phys. 163, 141-172, 1994

[4] Breitenlohner, P., Lavrelashvili, G. and Maison, D., preprint gr-qc/9703047

[5] Donats, E.E., Gal'tsov, D.V., and Zotov, M. Yu, preprint gr-qc/9612067

[6] Kunzle, H.P. and Masood-ul-Alam, A.K.M., J. Math. Phys. 31, 928-935, 1990

[7] Smoller, J., Wasserman, A., Commun. Math. Phys. 151, 303-325, 1993

[8] Smoller, J., Wasserman, A. and Yau, S.-T., Comm. Math. Phys. 154, 377-401, 1993

[9] Smoller, J. and Wasserman, A., preprint gr-qc/9706039, to appear in Comm. Math. Phys..

[10] Straumann, N. and Zhou, Z., Phys. Lett. B 237, 353-356, 1990

[11] Parker, T., and C. H. Taubes, Commun. Math. Phys. 84, 223-238, 1982

[12] Chandrasekhar, S., "The Mathematical Theory of Black Holes," Oxford University Press, 1992

[13] Finster, F., hep-th/9703083, J. Math. Phys. 39, No. 12, 6276-6290, 1998

[14] Smoller, J., "Shock Waves and Reaction-Diffusion Equations," 2nd ed., Springer, 1994

[15] Bjorken, J. and Drell, S., "Relativistic Quantum Mechanics," McGraw-Hill, 1964

[16] Adler, R., Bazin, M. and Schiffer, M., "Introduction to General Relativity," McGrawHill, 1975

[17] Sakurai, J.J., “Advanced Quantum Mechanics,” Addison-Wesley, 1967

Mathematics Department, Harvard University,

Cambridge, MA 02138 (FF \& STY)

email: felix@math.harvard.edu yau@math.harvard.edu smoller@umich.edu
Mathematics Department, The University of Michigan, Ann Arbor, MI 48109 